\title{
ON THE ONE-WAY SPECIFICATION PROPERTY AND LARGE DEVIATIONS FOR SYSTEMS WITH NON-DENSE ERGODIC MEASURES
}

\author{
Kenichiro YAMAMOTO \\ (Received 15 November 2016 and revised 11 March 2017)
}

\begin{abstract}
We introduce a weaker form of the specification property, called the 'one-way specification property', which holds for a broad class of systems whose ergodic measures are not dense in the set of invariant measures, including Smale's horseshoe map, DA map, and (symbolic spaces of) $(-\beta)$-transformations for Yrrap $\beta>1$. As an application, we show that these three examples satisfy a level-2 large deviation principle with the normalized Lebesgue measure.
\end{abstract}

\section{Introduction}

The specification property was introduced by Bowen in [1] to study the ergodic property of Axiom A diffeomorphisms. After that, several authors introduced various weaker forms of this property such as the weak specification property $[8, \mathbf{1 5}]$, the almost specification property $[\mathbf{1 9}, \mathbf{2 3}]$, the specification property for the collection of good words [5], and the non-uniform specification property [24] (see also [13] for details). In this paper, we introduce a new generalized version of the specification property and investigate large deviations for systems with this property.

Let $(X, d)$ be a compact metric space and let $f$ be a Borel measurable map from $X$ to itself. We denote by $\mathcal{M}(X)$ the set of all Borel probability measures on $X$ with the weak topology. We say that $(X, f)$ satisfies a level-2 large deviation principle with a reference measure $m \in \mathcal{M}(X)$ if there is an upper semicontinuous function $q: \mathcal{M}(X) \rightarrow[-\infty, 0]$, called a rate function, satisfying that

$$
\liminf _{n \rightarrow \infty} \frac{1}{n} \log m\left(\left\{x \in X: \frac{1}{n} \sum_{j=0}^{n-1} \delta_{f^{j}(x)} \in G\right\}\right) \geq \sup _{\mu \in G} q(\mu)
$$

holds for any open set $G \subset \mathcal{M}(X)$ and

$$
\limsup _{n \rightarrow \infty} \frac{1}{n} \log m\left(\left\{x \in X: \frac{1}{n} \sum_{j=0}^{n-1} \delta_{f^{j}(x)} \in F\right\}\right) \leq \sup _{\mu \in F} q(\mu)
$$

2010 Mathematics Subject Classification: Primary 37A50, 60F10, 37D35, 37B10.

Keywords: one-way specification property; large deviation principle; Smale's horseshoe map; DA map; $(-\beta)$-transformation. 
holds for any closed set $F \subset \mathcal{M}(X)$, where $\delta_{y}$ stands for the $\delta$-measure at the point $y \in X$. The present paper is largely motivated by the large deviations result of Young [26]. To state this result precisely, for $m \in \mathcal{M}(X)$ and a continuous function $\varphi$ on $X$, we consider the following condition:

$$
\begin{aligned}
& \lim _{\epsilon \rightarrow 0} \liminf _{n \rightarrow \infty} \inf _{x \in X}\left(\frac{1}{n} \log m\left(B_{n}(x, \epsilon)\right)+\frac{1}{n} \sum_{j=0}^{n-1} \varphi\left(f^{j}(x)\right)\right) \\
& =\lim _{\epsilon \rightarrow 0} \limsup _{n \rightarrow \infty} \sup _{x \in X}\left(\frac{1}{n} \log m\left(B_{n}(x, \epsilon)\right)+\frac{1}{n} \sum_{j=0}^{n-1} \varphi\left(f^{j}(x)\right)\right)=0 .
\end{aligned}
$$

Here $B_{n}(x, \epsilon):=\left\{y \in X: d\left(f^{j}(x), f^{j}(y)\right) \leq \epsilon, \quad 0 \leq j \leq n-1\right\}$. For dynamical systems with the specification property, Young essentially proved the following theorem.

TheOREM 1.1. [26, Theorem 1] Let $(X, d)$ be a compact metric space, let $f: X \rightarrow X$ be a continuous map such that the metric entropy map is upper semicontinuous, let $m \in \mathcal{M}(X)$ and $\varphi: X \rightarrow \mathbb{R}$ be a continuous function satisfying the condition (1.1). Suppose that $(X, f)$ satisfies the specification property (see Section 2.1 for the definition). Then $(X, f)$ satisfies a level-2 large deviation principle with $m$ and the rate function $q: \mathcal{M}(X) \rightarrow[-\infty, 0]$ is given by

$$
q(\mu)= \begin{cases}h(\mu)-\int \varphi d \mu & (\mu \text { is invariant }), \\ -\infty & (\text { otherwise }) .\end{cases}
$$

Here $h(\mu)$ denotes the metric entropy of $\mu$.

The specification property implies the 'entropy-density' of ergodic measures, that is, any invariant measure can be approximated by ergodic measures with similar entropies (see [18] for the precise definition). The entropy-density plays an important role in proving Theorem 1.1 (more precisely, to obtain the lower bounds). Indeed, there are many large deviations results for systems with the entropy-density such as $[\mathbf{3}, \mathbf{4}, \mathbf{6}, \mathbf{1 0}, \mathbf{1 8}, \mathbf{2 2}, \mathbf{2 5}, \mathbf{2 6}]$ and the authors of these papers use the entropy-density to get the lower bounds. However, there are also many important dynamical systems whose ergodic measures are not entropy-dense (even not dense). As far as we know, there are no resulting large deviations for such systems. So, the aim of this paper is to introduce a new generalized version of the specification property, called the 'one-way specification property', which holds for a large class of systems whose ergodic measures are not dense in the set of invariant measures, and prove the following generalization of Theorem 1.1.

THEOREM A. Let $(X, d)$ be a compact metric space, let $f: X \rightarrow X$ be a continuous map such that the metric entropy map is upper semicontinuous, let $m \in \mathcal{M}(X)$ and $\varphi: X \rightarrow \mathbb{R}$ be a continuous function satisfying the condition (1.1). Suppose that $(X, f)$ satisfies the oneway specification property (see Definition 2.2 ). Then $(X, f)$ satisfies a level-2 large deviation principle with $m$ and the rate function $q: \mathcal{M}(X) \rightarrow[-\infty, 0]$ is given by (1.2).

Remark 1.2. In Theorems A and 1.1, assumptions of $m$ and $\varphi$ are general but when we apply the theorems, $m$ is often chosen as a typical measure such as Lebesgue measure and maximal entropy measure. Moreover, the condition (1.1) states that the exponential decay of 
$m\left(B_{n}(x, \epsilon)\right)$ is equal to $-(1 / n) \sum_{j=0}^{n-1} \varphi\left(f^{j}(x)\right)$ for all $x \in X$ and so $m$ and $\varphi$ are closely related to each other. Thus, $\varphi$ is also an important function in many cases. Indeed, if $m$ is the Lebesgue measure, then one usually takes $\varphi$ as the logarithm of the absolute value of the Jacobian restricted to the unstable bundle and if $m$ is a maximal entropy measure, then $\varphi$ is usually taken as the constant function whose value is equal to the topological entropy.

In this paper, we give two applications of Theorem A. First, we apply Theorem A to Axiom A diffeomorphisms. Let $f$ be an Axiom A diffeomorphism of a $C^{\infty}$ closed manifold $X$. It is well known that the non-wandering set of $f$ can be decomposed to a finite union of basic sets $\Omega_{1} \cup \cdots \cup \Omega_{k}$ (see Section 2.4). Then, Theorem 1.1 is applicable to each subsystem $\left(\Omega_{i},\left.f\right|_{\Omega_{i}}\right)$ but in many cases, the whole system $(X, f)$ does not satisfy the specification property and so we cannot apply Theorem 1.1 to $(X, f)$. It is a novelty of this paper that Theorem A is applicable to the whole system $(X, f)$ for some classical Axiom A diffeomorphisms $f: X \rightarrow X$, such as Smale's horseshoe map and the DA map.

TheOREM B. Let $f: X \rightarrow X$ be a Smale's horseshoe map or a DA map given in Examples 3.2 and 3.3. Then $(X, f)$ satisfies a level-2 large deviation principle with the normalized Lebesgue measure on $X$, and the rate function $q: \mathcal{M}(X) \rightarrow[-\infty, 0]$ is given by (1.2) for some continuous function $\varphi$ on $X$ (see equations (6.1) and (6.2)).

The second application is a $(-\beta)$-transformation. The $(-\beta)$-transformation was recently introduced by Ito and Sadahiro in [12] as a natural modification of well-known $\beta$ transformations. For a real number $\beta>1$, the $(-\beta)$-transformation $T_{-\beta}$ is given by $x \mapsto-\beta x$ $(\bmod 1)$ and the $\beta$-transformation $T_{\beta}$ is given by $x \mapsto \beta x(\bmod 1)$ on the unit interval and so the definition of $T_{-\beta}$ is very similar to that of $T_{\beta}$. However, the dynamical property of $T_{-\beta}$ is different from that of $T_{\beta}$. For example, it is well known that $T_{\beta}$ is topologically mixing for all $\beta>1$, but we can easily find $\beta>1$ so that $T_{-\beta}$ is not transitive. Thus, it is worth considering the problems of $(-\beta)$-transformations, which are also considered in $\beta$-transformations. Recently, several authors studied the dynamical property of $(-\beta)$ transformations, such as Markovness and intrinsic ergodicity [7, 11, 14]. In this paper, we consider the large deviation principle for $(-\beta)$-transformations. In the case of $\beta$ transformations, the large deviation principle is well understood. Indeed, if the orbit of 1 under $T_{\beta}$ is eventually periodic, then the $\beta$-transformation satisfies the specification property and Theorem 1.1 is applicable. Moreover, Pfister and Sullivan [18] proved that for any $\beta>1$, the $\beta$-transformation satisfies a level-2 large deviation principle by using the entropy-density of ergodic measures. On the other hand, even if the orbit of 1 is eventually periodic under $T_{-\beta}$, the $(-\beta)$-transformation does not satisfy the specification property and its ergodic measures are not dense in general. Thus, neither Theorem 1.1 nor Pfister-Sullivan's result is applicable. In this paper, applying Theorem A, we obtain the large deviations result for $(-\beta)$-transformations under the condition that the orbit of 1 under $T_{-\beta}$ is eventually periodic.

THEOREM C. Let $T_{-\beta}:[0,1] \rightarrow[0,1]$ be a $(-\beta)$-transformation and suppose that the orbit of 1 under $T_{-\beta}$ is eventually periodic. Then $\left([0,1], T_{-\beta}\right)$ satisfies a level-2 large deviation principle with the Lebesgue measure on $[0,1]$ and the rate function $q: \mathcal{M}([0,1]) \rightarrow[-\infty, 0]$ is given by

$$
q(\mu)= \begin{cases}h(\mu)-\log \beta & (\mu \text { is invariant }) \\ -\infty & (\text { otherwise })\end{cases}
$$


Remark 1.3. We note that our definition of the $(-\beta)$-transformation is slightly different from the original [12] (see Remark 3.6).

A number $\beta>1$ with eventually periodic $T_{-\beta}$-orbit of 1 (in other words, $\beta>1$ to which Theorem $\mathrm{C}$ is applicable) is called a Yrrap number, which is studied deeply in [14]. It is not difficult to see that the set of Yrrap numbers is a countably infinite subset of $(1, \infty)$. The author thinks that we cannot apply Theorem A to most of non-Yrrap $\beta>1$. One remaining interesting question is to understand for which non-Yrrap $\beta>1\left([0,1], T_{-\beta}\right)$ satisfies a large deviation principle.

Though Theorem $\mathrm{C}$ is applicable only to countably many $\beta^{\prime}$, it has an interesting application. As mentioned above, Pfister and Sullivan [18] proved that for any $\beta>1$, the $\beta$ transformation satisfies a level-2 large deviation principle with a unique measure of maximal entropy and the rate function is given by (1.3). In this case, it is well known that the unique measure of maximal entropy is absolutely continuous with respect to the Lebesgue measure, with a density bounded above by $\beta /(\beta-1)$ and below by $(\beta-1) / \beta$ (see $[\mathbf{1 7}, \mathbf{2 0}]$ ). Thus, the $\beta$-transformation also satisfies a large deviation principle with the Lebesgue measure and the rate function is given by (1.3) as in the case of the measure of maximal entropy. However, a different phenomenon occurs in the case of $(-\beta)$-transformations. Indeed, as a consequence of Theorem $\mathrm{C}$, if $\beta$ is a minimal Pisot number, then the $(-\beta)$-transformation satisfies a level-2 large deviation principle with both the Lebesgue measure and the measure of maximal entropy, but these two rate functions do not coincide (see Example 6.6).

This paper is organized as follows. In Section 2, we establish our definitions. In Section 3, we give several examples of systems satisfying the one-way specification property, and give a proof of Theorem A in Section 4. In Section 5, we also give a proof of Corollary 1 which we state in Section 2.3. In Section 6, we apply our results to systems which appear in Section 3, in particular we give proofs of Theorems B and C.

\section{Preliminaries}

\subsection{Definitions, basic facts and lemmas}

Let $(X, d)$ be a compact metric space and let $f: X \rightarrow X$ be a continuous map. We denote by $C(X)$ the Banach space of continuous real-valued functions of $X$ with the sup norm $\|\cdot\|_{\infty}$ and by $\mathcal{M}(X)$ the set of all Borel probability measures on $X$ with the weak topology. Since $C(X)$ is separable, there exists a countable set $\left\{\varphi_{1}, \varphi_{2}, \ldots\right\}$ which is dense in $C(X)$ and $\left\|\varphi_{n}\right\|_{\infty} \neq 0$ holds for any $n \geq 1$. For $\mu, v \in \mathcal{M}(X)$, we define

$$
D(\mu, \nu):=\sum_{n=1}^{\infty} \frac{\left|\int \varphi_{n} d \mu-\int \varphi_{n} d \nu\right|}{2^{n+1}\left\|\varphi_{n}\right\|_{\infty}} .
$$

Then $D$ is a compatible metric for $\mathcal{M}(X)$. By an easy calculation, we have the following lemma. 
LEMMA 2.1 .

(1) $D(\mu, v) \leq 1$ holds for any $\mu, v \in \mathcal{M}(X)$.

(2) Let $\sum_{i=1}^{p} a_{i} \mu_{i}$ and $\sum_{i=1}^{p} a_{i} \nu_{i}$ be finite convex combinations of Borel probability measures on $X$. Then we have

$$
D\left(\sum_{i=1}^{p} a_{i} \mu_{i}, \sum_{i=1}^{p} a_{i} v_{i}\right) \leq \sum_{i=1}^{p} a_{i} D\left(\mu_{i}, v_{i}\right) .
$$

(3) Let $\sum_{i=1}^{p} a_{i} \mu_{i}$ and $\sum_{i=1}^{p} b_{i} v_{i}$ be finite convex combinations of Borel probability measures on $X$. Suppose that $\sum_{i=1}^{p}\left|a_{i}-b_{i}\right| \leq \zeta$ and $D\left(\mu_{i}, v_{i}\right) \leq \zeta$ for $1 \leq i \leq p$.

Then we have

$$
D\left(\sum_{i=1}^{p} a_{i} \mu_{i}, \sum_{i=1}^{p} b_{i} v_{i}\right) \leq 2 \zeta .
$$

Let $\mathcal{M}_{f}(X) \subset \mathcal{M}(X)$ be the set of all $f$-invariant Borel probability measures, and let $\mathcal{M}_{f}^{e}(X) \subset \mathcal{M}_{f}(X)$ be the set of all ergodic measures. For $n \geq 1$, we define $\mathcal{E}_{n}: X \rightarrow \mathcal{M}(X)$ by $\mathcal{E}_{n}(x):=(1 / n) \sum_{j=0}^{n-1} \delta_{f^{j}(x)}$.

Before giving a definition of the one-way specification property, we recall the definition of the specification property. We say that $(X, f)$ satisfies the specification property if for any $\epsilon>0$, there exists an integer $M \geq 0$ such that for any $x_{1}, \ldots, x_{k} \in X$ and any $n_{1}, \ldots, n_{k} \in \mathbb{N}$ there exists $y \in X$ and integers $0 \leq M_{1}, \ldots, M_{k-1} \leq M$ such that $d\left(f^{i}\left(x_{j}\right), f^{i+\sum_{t=1}^{j-1}\left(n_{t}+M_{t}\right)}(y)\right) \leq \epsilon$ holds for $0 \leq i \leq n_{j}-1$ and $1 \leq j \leq k$. Here we set $\sum_{t=1}^{0}\left(n_{t}+M_{t}\right):=0$. (Let us remark that the above definition of the specification property is slightly weaker than the original [1].) Roughly speaking, the specification property guarantees the existence of an orbit which traces all specified orbit-segments. The one-way specification property, defined below, guarantees the existence of an orbit which traces specified orbitsegments 'contained in the non-wandering set.' A point $x \in X$ is said to be non-wandering if for every neighborhood $U$ of $x$, there exists an integer $n>0$ such that $U \cap f^{-n}(U) \neq \emptyset$. The set of all non-wandering points is called the non-wandering set and is denoted by $\Omega(f)$. It is well known that $\mu(\Omega(f))=1$ for any $\mu \in \mathcal{M}_{f}(X)$ (see [9, Proposition 6.19] for instance). We then define the main property of this paper.

Definition 2.2. We say that $(X, f)$ satisfies the one-way specification property if there exist compact subsets $X_{1}, \ldots, X_{q}$ of $X$ (which are not necessarily disjoint) such that:

(1) $f\left(X_{i}\right) \subset X_{i}$ for $1 \leq i \leq q$;

(2) $\Omega(f)=\bigcup_{i=1}^{q} X_{i}$; and

(3) for any $\epsilon>0$, there exists an integer $M \geq 0$ such that for any $x_{1} \in X_{i(1)}, \ldots, x_{k} \in$ $X_{i(k)}$ with $i(1) \leq \cdots \leq i(k)$ and any $n_{1}, \ldots, n_{k} \in \mathbb{N}$, there exist $y \in X$ and integers $0 \leq M_{1}, \ldots, M_{k-1} \leq M$ such that

$$
d\left(f^{i}\left(x_{j}\right), f^{i+\sum_{t=1}^{j-1}\left(n_{t}+M_{t}\right)}(y)\right) \leq \epsilon \quad\left(0 \leq i \leq n_{j}-1,1 \leq j \leq k\right) .
$$

Here we set $\sum_{i=1}^{0}\left(n_{t}+M_{t}\right):=0$.

The sense of 'one-way' in the above definitions seems to be well understood once we give examples in Section 3. We say that $f$ is transitive if there is a dense orbit. It is easy to see that if $f$ satisfies the specification property, then $f$ is transitive. However, the one-way 
specification property defined above does not imply transitivity in general (see Section 3). We sometimes say that $(X, f)$ satisfies the one-way specification property 'with $\left\{X_{1}, \ldots, X_{q}\right\}$.' We note that if $(X, f)$ satisfies the one-way specification property with $\left\{X_{1}, \ldots, X_{q}\right\}$, then every ergodic measure is supported on some $X_{i}(1 \leq i \leq q)$. In fact, the following lemma holds.

Lemma 2.3. Let $\mu \in \mathcal{M}_{f}^{e}(X)$ and let $X_{1}, \ldots, X_{q}$ be compact subsets of $X$. Suppose that $f\left(X_{i}\right) \subset X_{i}$ for $1 \leq i \leq q$ and $\Omega(f)=\bigcup_{i=1}^{q} X_{i}$. Then there exists an integer $1 \leq i \leq q$ such that $\mu\left(X_{i}\right)=1$.

Proof. Since $\mu(\Omega(f))=1$, there exists an integer $1 \leq i \leq q$ such that $\mu\left(X_{i}\right)>0$. Let $G_{\mu}$ be the set of all generic points for $\mu$, that is

$$
G_{\mu}=\left\{x \in X: \lim _{n \rightarrow \infty} \mathcal{E}_{n}(x)=\mu\right\}
$$

(see [9, p. 21]). Since $\mu$ is ergodic, we have $\mu\left(G_{\mu}\right)=1$, and which implies that $\mu\left(G_{\mu} \cap\right.$ $\left.X_{i}\right)>0$. In particular, $G_{\mu} \cap X_{i} \neq \emptyset$.

Let us take a point $x \in G_{\mu} \cap X_{i}$. Then it follows from $f\left(X_{i}\right) \subset X_{i}$ that $f^{n}(x) \in X_{i}$ for $n \geq 0$, which implies $\left(\mathcal{E}_{n}(x)\right)\left(X_{i}\right)=1$ for any $n \geq 1$. Since $\lim _{n \rightarrow \infty} \mathcal{E}_{n}(x)=\mu$ and $X_{i}$ is compact, we have

$$
\mu\left(X_{i}\right) \geq \limsup _{n \rightarrow \infty}\left(\mathcal{E}_{n}(x)\right)\left(X_{i}\right)=1,
$$

which proves the lemma.

Let $\epsilon>0$ and $n \geq 1$. A subset $E \subset X$ is called $(n, \epsilon)$-separated if for any two distinct points $x, y \in E$, there exists $0 \leq j \leq n-1$ such that $d\left(f^{j}(x), f^{j}(y)\right)>\epsilon$. To show Theorem A we use the following result, which is derived by a tiny modification of $[\mathbf{1 8}$, Proposition 2.1].

Proposition 2.4. For any $\mu \in \mathcal{M}_{f}^{\mathrm{e}}(X)$ and any $h<h(\mu)$, there exists an $\epsilon>0$ such that for any neighborhood $F$ of $\mu$ there exists an integer $N>0$ such that for any $n \geq N$ and for any Borel subset $B \subset X$ with $\mu(B)=1$, there exists an $(n, \epsilon)$-separated set $\Gamma \subset \mathcal{E}_{n}^{-1}(F) \cap B$ such that $\sharp \Gamma \geq e^{n h}$.

\subsection{Symbolic dynamics}

Let $A$ be a finite set and let $A^{\mathbb{Z}_{+}}(\mathbb{Z}:=\mathbb{N} \cup\{0\})$ be the set of all one-sided infinite sequences on the alphabet $A$, endowed with the standard metric $d\left(\omega, \omega^{\prime}\right)=2^{-t\left(\omega, \omega^{\prime}\right)}$, where $t\left(\omega, \omega^{\prime}\right)=$ $\min \left\{k: \omega_{k} \neq \omega_{k}^{\prime}\right\}$. The shift map $\sigma: A^{\mathbb{Z}_{+}} \rightarrow A^{\mathbb{Z}_{+}}$is defined by $(\sigma(\omega))_{i}=\omega_{i+1}$ for $i \geq 0$. We say that a compact subset $X \subset A^{\mathbb{Z}_{+}}$is a subshift if $\sigma(X) \subset X$ holds. We often denote $\sigma$ instead of the restriction map $\left.\sigma\right|_{X}$ if no confusion arises. The language of $X$, denoted by $\mathcal{L}=\mathcal{L}(X)$, is the set of all finite words that appear in any sequence $\omega \in X$, that is,

$$
\mathcal{L}(X):=\left\{w \in A^{*}:[w] \neq \emptyset\right\},
$$

where $A^{*}=\bigcup_{n \geq 0} A^{n}$ and $[w]=\left\{a \in X: a_{0} \cdots a_{n-1}=w\right\}$ is a cylinder set for $w \in A^{n}$. Given $w \in \mathcal{L}(X)$, let $|w|$ denote the length of $w$, and set $\mathcal{L}_{n}(X)=\{w \in \mathcal{L}(X):|w|=n\}$ for $n \geq 1$. By our choice of the metric $d$ on $A^{\mathbb{Z}_{+}}$, we have the following proposition. 
Proposition 2.5. Let $(X, \sigma)$ be a subshift on a finite alphabet.

(1) $(X, \sigma)$ satisfies the specification property if and only if there exists an integer $M>0$ such that for any $w_{1}, \ldots, w_{k} \in \mathcal{L}(X)$, there exist $v_{1}, \ldots, v_{k-1} \in \mathcal{L}(X)$ such that $\left|v_{i}\right| \leq M$ for $1 \leq i \leq k-1$ and $w_{1} v_{1} w_{2} v_{2}, \ldots, w_{k-1} v_{k-1} w_{k} \in \mathcal{L}(X)$.

(2) $(X, \sigma)$ satisfies the one-way specification property if and only if there exist subshifts $X_{1}, \ldots, X_{q} \subset X$ and an integer $M \geq 0$ such that $\Omega\left(\left.\sigma\right|_{X}\right)=$ $\bigcup_{i=1}^{q} \Omega\left(\left.\sigma\right|_{X_{i}}\right)$, and for any $w_{1} \in \mathcal{L}\left(X_{i(1)}\right), \ldots, w_{k} \in \mathcal{L}\left(X_{i(k)}\right)$ with $i(1) \leq \cdots \leq$ $i(k)$, there exist $v_{1}, \ldots, v_{k-1} \in \mathcal{L}(X)$ such that $\left|v_{i}\right| \leq M$ for $1 \leq i \leq k-1$ and $w_{1} v_{1} w_{2} v_{2}, \ldots, w_{k-1} v_{k-1} w_{k} \in \mathcal{L}(X)$.

(3) $[\mathbf{1 8}$, Proposition 4,2] Let $m \in \mathcal{M}(X)$ and $\varphi \in C(X)$. Then $\varphi$ satisfies the condition (1.1) if and only if

$$
\begin{aligned}
& \liminf _{n \rightarrow \infty} \inf _{w \in \mathcal{L}_{n}(X)}\left(\frac{1}{n} \log m([w])+\inf _{x \in[w]} \frac{1}{n} \sum_{j=0}^{n-1} \varphi\left(\sigma^{j}(x)\right)\right) \\
& \quad=\limsup _{n \rightarrow \infty} \sup _{w \in \mathcal{L}_{n}(X)}\left(\frac{1}{n} \log m([w])+\sup _{x \in[w]} \frac{1}{n} \sum_{j=0}^{n-1} \varphi\left(\sigma^{j}(x)\right)\right)=0 .
\end{aligned}
$$

\subsection{Symbolic spaces of piecewise expanding interval systems}

Let $I=[0,1]$ be the unit interval, and let $f: I \rightarrow I$ be such that there exist $p \geq 2$ and $0=a_{0}<a_{1}<\cdots<a_{p}=1$ such that writing $I_{j}=\left(a_{j}, a_{j+1}\right)$, the restriction $\left.f\right|_{I_{j}}$ is $C^{1}$ and satisfies $\alpha \leq\left|f^{\prime}(x)\right| \leq \beta\left(x \in \bigcup_{j=1}^{p-1} I_{j}\right)$ for some $1<\alpha \leq \beta$. We say that $(I, f)$ is a piecewise expanding interval system.

Let $A=\{0, \ldots, p-1\}, S=\left\{a_{0}, \ldots, a_{p}\right\}$ and $I^{\prime}=I \backslash \bigcup_{i \geq 0} f^{-i}(S)$. We define the map $i^{\prime}: I^{\prime} \rightarrow A^{\mathbb{Z}_{+}}$by $\left(i^{\prime}(x)\right)_{k}=j$ if $f^{k}(x) \in I_{j}$. We note that $i^{\prime}$ is well defined and injective on $I^{\prime}$ since $\left|f^{\prime}(x)\right| \geq \alpha>1$ for all $x \in I \backslash S$. We set $\Sigma_{f}=\operatorname{cl}\left\{i^{\prime}\left(I^{\prime}\right)\right\}$, where $\operatorname{cl}(A)$ denotes the closure of the set $A$. Then $\left(\Sigma_{f}, \sigma\right)$ is a subshift. We call $\left(\Sigma_{f}, \sigma\right)$ the symbolic space of $(I, f)$.

We define $\Psi: \Sigma_{f} \rightarrow I$ by $\Psi(\omega)=\bigcap_{k=0}^{\infty} f^{-k} \operatorname{cl}\left(I_{\omega_{k}}\right)$. It is well known that $(I, f)$ is a topological factor of $\left(\Sigma_{f}, \sigma\right)$ with $\Psi$ as a factor map. To prove Theorem C, we use the following proposition, which is derived from Theorem A.

COROLlary 1. Let $([0,1], f)$ be a piecewise expanding interval system, let $\left(\Sigma_{f}, \sigma\right)$ be a symbolic space of $([0,1], f)$, let $m$ be the Lebesgue measure on $[0,1]$ and let $\varphi:[0,1] \rightarrow \mathbb{R}$ be a continuous function. Suppose that the three conditions (I)-(III) hold.

(I) There exists a measurable map $\Phi:[0,1] \rightarrow \Sigma_{f}$ such that $\Psi \circ \Phi:[0,1] \rightarrow[0,1]$ is an identity map and $\Phi \circ f=\sigma \circ \Phi$. Moreover, there exists a Borel set $Y \subset \Sigma_{f}$ such that $[0,1] \backslash \Psi(Y)$ is countable and $\Phi \circ \Psi: Y \rightarrow \Phi(\Psi(Y))$ is an identity map.

(II) $m_{f}:=m \circ \Phi^{-1}$ and $\varphi \circ \Psi$ satisfy the condition (1.1).

(III) $\left(\Sigma_{f}, \sigma\right)$ satisfies the one-way specification property.

Here $\Psi: \Sigma_{f} \rightarrow[0,1]$ is the factor map defined above. Then $([0,1], f)$ satisfies a level-2 large deviation principle with $m$ and the rate function $q: \mathcal{M}([0,1]) \rightarrow[-\infty, 0]$ is given by (1.2).

We give a proof of Corollary 1 in Section 5. 


\subsection{Axiom A diffeomorphisms}

Let $M$ be a $C^{\infty}$ closed manifold with a Riemannian metric $\|\cdot\|$ on the tangent bundle $T M$ and let $f: M \rightarrow M$ be a diffeomorphism. We say that a closed subset $\Lambda \subset M$ is hyperbolic if $f(\Lambda)=\Lambda$ and the tangent bundle $\left.T M\right|_{\Lambda}$ splits into the $D f$-invariant direct sum $\left.T M\right|_{\Lambda}=$ $E^{s} \oplus E^{u}$ of sub-bundles $E^{s}$ and $E^{u}$, and there are constants $C>0$ and $0<\lambda<1$ such that $\left\|\left.D_{x} f^{n}\right|_{E^{s}}\right\| \leq C \lambda^{n}$ and $\left\|\left.D_{x} f^{-n}\right|_{E^{u}}\right\| \leq C \lambda^{n}$ for $n \geq 0$ holds. We say that $f$ satisfies Axiom A if $\Omega(f)$ is hyperbolic and $\Omega(f)=\operatorname{cl}\left(\left\{x \in M: f^{n}(x)=x\right.\right.$ for some $\left.\left.n \geq 1\right\}\right)$. It is well known that if $f$ satisfies Axiom A, then there exist pairwise disjoint closed sets $\Omega_{1}, \ldots, \Omega_{k}$ such that $\Omega(f)=\bigcup_{i=1}^{k} \Omega_{i}, f\left(\Omega_{i}\right)=\Omega_{i}$ and $\left.f\right|_{\Omega_{i}}$ is transitive for $1 \leq i \leq k$ (see [2, (3.5)] for instance). We call each $\Omega_{i}$ the basic set. As mentioned in Section 2.1, our definition of the specification property is slightly weaker than the original one introduced by Bowen and it is known that an Axiom A diffeomorphism restricted to the basic set does not satisfy the stronger variant of the specification property in the sense Bowen. But 'in our case', it satisfies the specification property.

Lemma 2.6. Let $f$ be an Axiom A diffeomorphism and let $\Lambda$ be a basic set. Then $(\Lambda, f)$ satisfies the specification property.

Proof. It follows from [1, (2.7) and (2.10)] that there exist pairwise disjoint closed sets $\Lambda_{1}, \ldots, \Lambda_{m}$ such that $\Lambda=\bigcup_{j=1}^{m} \Lambda_{m}, f\left(\Lambda_{j}\right)=\Lambda_{j+1}$ for $1 \leq j \leq m-1, f\left(\Lambda_{m}\right)=\Lambda_{1}$, and $\left(\Lambda_{1}, f^{m}\right)$ satisfies the stronger variant of the specification property. More precisely, for any $\delta>0$, there exists an integer $M(\delta)>0$ such that for any $x_{1}, \ldots, x_{k} \in \Lambda_{1}$ and any $n_{1}, \ldots, n_{k} \in \mathbb{N}$, we can find a point $y \in \Lambda_{1}$ such that $d\left(f^{m i}\left(x_{j}\right), f^{m\left(i+\sum_{t=1}^{j-1}\left(n_{t}+M(\delta)\right)\right)}(y)\right) \leq$ $\delta$ holds for $0 \leq i \leq n_{j-1}$ and $1 \leq j \leq k$. Here we set $\sum_{t=1}^{0}\left(n_{t}+M(\delta)\right):=0$.

Given $\epsilon>0$, by the uniform continuity of $f$ on $\Lambda$, we can find $\delta>0$ such that $d\left(f^{j}(x), f^{j}(y)\right) \leq \epsilon$ for $0 \leq j \leq m-1$ whenever $d(x, y) \leq \delta$. Take any $x_{1}, \ldots, x_{k}$ and $n_{1}, \ldots, n_{k} \in \mathbb{N}$. For each $1 \leq j \leq k$, choose $0 \leq a_{j}, b_{j} \leq m-1$ so that $f^{-a_{j}}\left(x_{j}\right), f^{n_{j}+b_{j}}\left(x_{j}\right) \in \Lambda_{1}$ holds. For notational simplicity, we set $x_{j}^{\prime}:=f^{-a_{j}}\left(x_{j}\right)$ and choose $n_{j}^{\prime}$ so that $f^{m\left(n_{j}^{\prime}-1\right)}\left(x_{j}^{\prime}\right)=f^{n_{j}+b_{j}}\left(x_{j}\right)$ holds for $1 \leq j \leq k$. Let $M(\delta)$ be as above. Then we can find a point $y^{\prime} \in \Lambda_{i}$ such that $d\left(f^{m i}\left(x_{j}^{\prime}\right), f^{m\left(i+\sum_{t=1}^{j-1}\left(n_{t}^{\prime}+M(\delta)\right)\right)}\left(y^{\prime}\right)\right) \leq \delta$ holds for $0 \leq i \leq n_{j}^{\prime}-1$ and $1 \leq j \leq k$, where we set $\sum_{t=1}^{0}\left(n_{t}^{\prime}+M(\delta)\right):=0$. By our choice of $\delta>0$, this implies that $d\left(f^{i}\left(x_{j}\right), f^{i+\sum_{t=1}^{j-1}\left(n_{t}+M_{t}\right)}(y)\right) \leq \epsilon$ for $0 \leq i \leq n_{j}-1$ and $1 \leq$ $j \leq k$. Here we set $y:=f^{a_{1}}\left(y^{\prime}\right), M_{t}:=m(M(\delta)+1)+b_{t}+a_{t+1}$ for $1 \leq t \leq k-1$, and $\sum_{t=1}^{0}\left(n_{t}+M_{t}\right):=0$. This implies that $(\Lambda, f)$ satisfies the specification property.

\section{Examples}

\subsection{Simple symbolic example}

In this subsection, we give a simple example of a symbolic system which satisfies the oneway specification property.

Example 3.1. We consider a piecewise expanding interval system whose symbolic space has the one-way specification property. Let $I=[0,1]$ be the unit interval and define $f: I \rightarrow I$ 


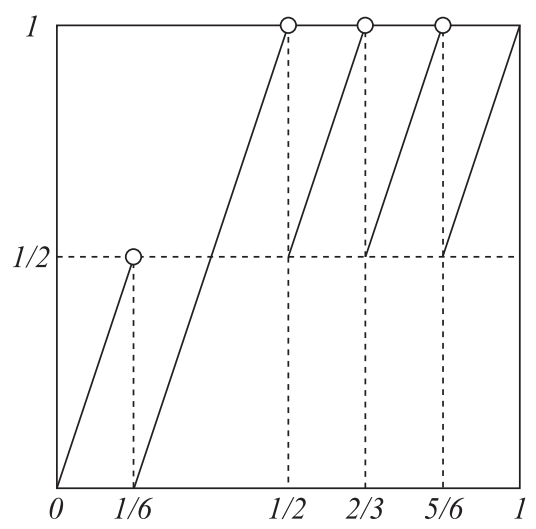

FIGURE 1. A graph of $f$.

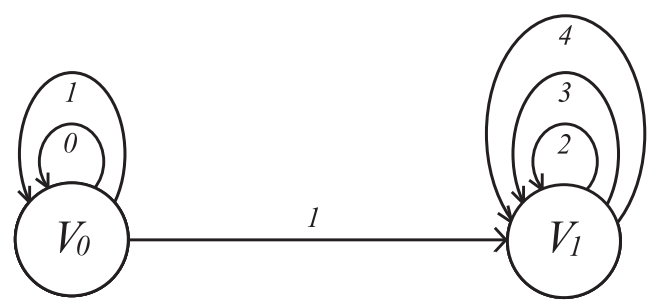

Figure 2. A graph $\Gamma$.

by

$$
f(x)= \begin{cases}3 x & \left(0 \leq x<\frac{1}{6}\right) \\ 3 x-\frac{1}{2} & \left(\frac{1}{6} \leq x<\frac{1}{2}\right) \\ 3 x-1 & \left(\frac{1}{2} \leq x<\frac{2}{3}\right) \\ 3 x-\frac{3}{2} & \left(\frac{2}{3} \leq x<\frac{5}{6}\right) \\ 3 x-2 & \left(\frac{5}{6} \leq x \leq 1\right)\end{cases}
$$

(A graph of $f$ is sketched in Figure 1.) We set $I_{0}:=\left(0, \frac{1}{6}\right), I_{1}:=\left(\frac{1}{6}, \frac{1}{2}\right), I_{2}:=\left(\frac{1}{2}, \frac{2}{3}\right)$, $I_{3}:=\left(\frac{2}{3}, \frac{5}{6}\right), I_{4}:=\left(\frac{5}{6}, 1\right)$, and let $\left(\Sigma_{f}, \sigma\right)$ be the symbolic space of $(I, f)$ defined as in Section 2.3. Then it is not difficult to see that the language of $\Sigma_{f}$ is the set of all subwords of the form $w 1 v\left(w \in\{0,1\}^{\mathbb{Z}_{+}}, v \in\{2,3,4\}^{\mathbb{Z}_{+}}\right)$. In other words, $\Sigma_{f}$ is the set of all infinite paths started from some vertex of a Graph $\Gamma$, which is shown in Figure 2. Thus, $\left(\Sigma_{f}, \sigma\right)$ satisfies the one-way specification property with $\left\{\{0,1\}^{\mathbb{Z}_{+}},\{2,3,4\}^{\mathbb{Z}_{+}}\right\}$. 


\subsection{Axiom A diffeomorphisms}

In this subsection, we consider two well-known Axiom A diffeomorphisms, Smale's horseshoe map and the DA map, and prove that these systems satisfy the one-way specification property.

Example 3.2. Let $S^{2}=\mathbb{R} \cup\left\{p_{\infty}\right\}$ be a 2 -sphere, where $p_{\infty}$ denotes the point at infinity. Given $0 \leq a_{1}<b_{1}<a_{2}<b_{2} \leq 1$ and $0 \leq c_{1}<d_{1}<c_{2}<d_{2} \leq 1$, we define $H_{j}:=[0,1] \times$ $\left[a_{j}, b_{j}\right]$ and $V_{j}:=\left[c_{j}, d_{j}\right] \times[0,1]$ for $j=1,2$ and $G:=[0,1] \times\left(a_{2}, b_{1}\right)$. We also set $S:=[0,1] \times[0,1], A:=\left\{(x, y):\left(x-\frac{1}{2}\right)^{2}+y^{2} \leq \frac{1}{4}, y \leq 0\right\}, B:=\left\{(x, y):\left(x-\frac{1}{2}\right)^{2}+\right.$ $\left.(y-1)^{2} \leq \frac{1}{4}, y \geq 1\right\}$ and $N:=S \cup A \cup B$. Then it is well known that one can construct a $C^{\infty}$ Axiom A diffeomorphism $f: S^{2} \rightarrow S^{2}$, called 'Smale's horseshoe map', satisfying the following properties. (We refer to [21, Section 8.4] for construction and details.)

(S1) $f\left(H_{j}\right)=V_{j}$ for $j=1,2$ and $S \cap f^{-1}(S)=H_{1} \cup H_{2}$.

(S2) There are constants $0<\lambda_{s}<\frac{1}{2}$ and $\lambda_{u}>2$ such that $D_{x} f=\left(\begin{array}{cc}a_{x} & 0 \\ 0 & b_{x}\end{array}\right),\left|a_{x}\right|=\lambda_{s}$ and $\left|b_{x}\right|=\lambda_{u}$ hold for any $x \in H_{1} \cup H_{2}$.

(S3) $f(N) \subset \operatorname{int}(N), f(G) \subset B$ and $f(A \cup B) \subset \operatorname{int}(A)$ hold, where $\operatorname{int}(Y)$ denotes the interior of $Y$.

(S4) $f$ is a contraction mapping on $A$. In particular, there is a unique fixed point sink $p_{0} \in A$.

(S5) $p_{\infty}$ is a fixed point source.

(S6) Let $\Lambda:=\bigcap_{j=-\infty}^{\infty} f^{j}(S)$. Then $\Omega(f)=\left\{p_{\infty}\right\} \cup \Lambda \cup\left\{p_{0}\right\}$ holds and $\Lambda$ is a basic set. (So by Lemma 2.6, $(\Lambda, f)$ satisfies the specification property.)

In Figure 3, we sketch subsets $N$ and $f(N)$.
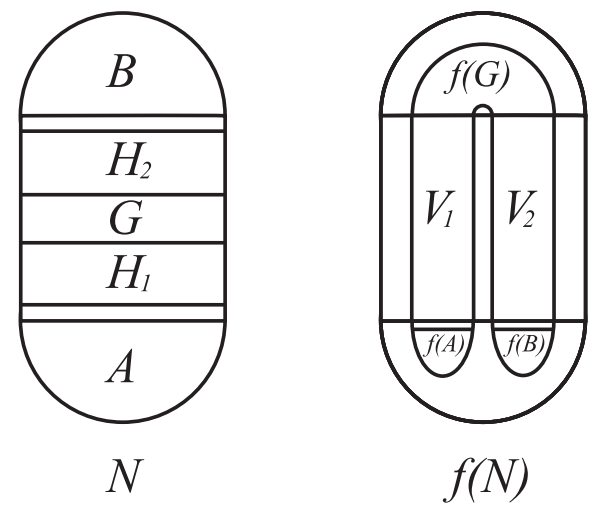

FIGURE 3. Sketches of subsets $N$ and $f(N)$.

In what follows we will show that $\left(S^{2}, f\right)$ satisfies the one-way specification property with $\left\{\left\{p_{\infty}\right\}, \Lambda,\left\{p_{0}\right\}\right\}$. First, by (S1)-(S4), it is not difficult to see that for any $\epsilon>0$, there is an integer $M_{1}>0$ such that for any $x \in \Lambda$ and any $n \geq 1$, we can find $y \in B_{n}(x, \epsilon)$ such that $f^{-M_{1}}(y) \in S^{2} \backslash N$ and $f^{n+M_{1}}(y) \in A$ hold. Since $\Omega(f)=\left\{p_{\infty}\right\} \cup \Lambda \cup\left\{p_{0}\right\}$ and $p_{\infty}$ is a source, for any $\epsilon>0$, there exists an integer $M_{2}>0$ such that for any $x \in S^{2} \backslash N$ and any $n \geq M_{2}, d\left(f^{-n}(x), p_{\infty}\right) \leq \epsilon$ holds. Moreover, it follows from (S5) that for any $\epsilon$, we can find an integer $M_{3}>0$ such that for any $x \in A$ and any $n \geq M_{3}, d\left(f^{n}(x), p_{0}\right) \leq \epsilon$ holds. These together with (S6) imply that $\left(S^{2}, f\right)$ satisfies the one-way specification property with $\left\{\left\{p_{\infty}\right\}, \Lambda,\left\{p_{0}\right\}\right\}$. 
Example 3.3. Let $\mathbb{T}^{2}$ be a two-dimensional torus. Then it is known that there is a $C^{\infty}$ Axiom A diffeomorphism $f: \mathbb{T}^{2} \rightarrow \mathbb{T}^{2}$, called the 'DA map', satisfying the following properties.

(D1) $\Omega(f)=\left\{p_{0}\right\} \cup \Lambda$, where $p_{0}$ is a fixed point source and $\Lambda$ is a hyperbolic basic set with one-dimensional unstable bundle $E^{u}$.

(D2) For any $\epsilon>0$, we can find an integer $N \geq 0$ such that for any $x \in \Lambda, W_{\epsilon}^{s}(x) \cap$ $f^{N}\left(W_{\epsilon}^{u}\left(p_{0}\right)\right) \neq \emptyset$. Here $W_{\epsilon}^{u}\left(p_{0}\right):=\left\{y \in \mathbb{T}^{2}: d\left(f^{-n}(y), f^{-n}\left(p_{0}\right)\right) \leq \epsilon, \quad n \geq 0\right\}$ and $W_{\epsilon}^{s}(x):=\left\{y \in \mathbb{T}^{2}: d\left(f^{n}(x), f^{n}(y)\right) \leq \epsilon, n \geq 0\right\}$.

(D3) There exists a small open neighborhood $U$ of $p_{0}$ such that $\Lambda=\bigcap_{j=0}^{\infty} f^{j}\left(\mathbb{T}^{2} \backslash U\right)$. Moreover, $f$ is expanding on $U$, that is, there exists a constant $C>0$ and $0<\lambda<1$ so that $\left\|D_{x} f^{-n}\right\| \leq C \lambda^{n}$ holds for $x \in U$ and $n \geq 0$.

Such a diffeomorphism can be obtained by modifying a hyperbolic toral automorphism in a small neighborhood of the origin. See [21, Section 8.8] for details.

Now we prove that $\left(\mathbb{T}^{2}, f\right)$ satisfies the one-way specification property with $\left\{\left\{p_{0}\right\}, \Lambda\right\}$. By Lemma 2.6, $(\Lambda, f)$ satisfies the specification property. Take any $\epsilon>0$ and let $M>0$ be an integer as in the definition of the specification property. Take $x_{1}=p_{0}, x_{2}, \ldots, x_{k} \in \Lambda$ and $n_{1}, \ldots, n_{k} \in \mathbb{N}$. Since $(\Lambda, f)$ satisfies the specification property, we can find $y^{\prime} \in$ $\Lambda$ and integers $0 \leq M_{2}, \ldots, M_{k-1} \leq M$ such that $d\left(f^{i}\left(x_{j}\right), f^{i+\sum_{t=2}^{j-1}\left(n_{t}+M_{t}\right)}\left(y^{\prime}\right)\right) \leq \epsilon$ for $0 \leq i \leq n_{j}-1$ and $2 \leq j \leq k$. Then it follows from (D2) that we can find $N \in \mathbb{N}$ such that $W_{\epsilon}^{s}\left(y^{\prime}\right) \cap f^{N}\left(W_{\epsilon}^{u}\left(p_{0}\right)\right) \neq \emptyset$. Take a point $y^{\prime \prime} \in W_{\epsilon}^{s}\left(y^{\prime}\right) \cap f^{N}\left(W_{\epsilon}^{u}\left(p_{0}\right)\right)$, and set $y:=$ $f^{-N-n_{1}}\left(y^{\prime \prime}\right)$ and $M_{1}:=N$. Then we have $d\left(f^{i}\left(x_{j}\right), f^{i+\sum_{t=1}^{j-1}\left(n_{t}+M_{t}\right)}(y)\right) \leq 2 \epsilon$ for $0 \leq i \leq$ $n_{j}-1$ and $1 \leq j \leq k$, which implies that $\left(\mathbb{T}^{2}, f\right)$ satisfies the one-way specification property with $\left\{\left\{p_{0}\right\}, \Lambda\right\}$.

\section{3. (- $\beta)$-transformations}

For $\beta>1$, we define a $(-\beta)$-transformation $T_{-\beta}:[0,1] \rightarrow[0,1]$ as follows. Let $b:=$ $\max \{k \in \mathbb{Z}: k<\beta\}$ and set $I_{i}:=(i / \beta,(i+1) / \beta)$ for $0 \leq i \leq b-1$ and $I_{b}:=(b / \beta, 1)$. First, we define values of $T_{-\beta}$ on $\bigcup_{i=0}^{b} I_{b}$ by

$$
T_{-\beta}(x):=-\beta x+(i+1) \quad(0 \leq i \leq b) .
$$

Then, for $x \notin \bigcup_{i=0}^{\infty} T_{-\beta}^{-i}(\{0,1 / \beta, \ldots, b / \beta, 1\})$, one can define $i^{\prime}(x) \in\{0,1, \ldots, b\}^{\mathbb{Z}_{+}}$by $\left(i^{\prime}(x)\right)_{k}=j$ if $T_{-\beta}^{k}(x) \in I_{j}$. We set $i^{\prime}(1):=\lim _{x \rightarrow 1-0} i^{\prime}(x)$.

In what follows we will define values of $T_{-\beta}$ on $\{0,1 / \beta, \ldots, b / \beta, 1\}$.

Case 1. $i^{\prime}(1)$ is of the form $(w 0)^{\infty}=w 0 w 0 w 0 \cdots$ for some $w \in\{0,1, \ldots, b\}^{*}$. In this case, we define $T_{-\beta}$ on $\{0,1 / \beta, \ldots, b / \beta, 1\}$ by

$$
T_{-\beta}(x)= \begin{cases}1 & (x=0), \\ 0 & (x=1 / \beta, \ldots, b / \beta), \\ \lim _{x \rightarrow 1-0} T_{-\beta}(x) & (x=1) .\end{cases}
$$


Case 2. $i^{\prime}(1)$ is not of the form $(w 0)^{\infty}=w 0 w 0 w 0 \cdots$. In this case, we define $T_{-\beta}$ on $\{0,1 / \beta, \ldots, b / \beta, 1\}$ by

$$
T_{-\beta}(x)= \begin{cases}1 & (x=0), \\ 1 & (x=1 / \beta, \ldots, b / \beta), \\ \lim _{x \rightarrow 1-0} T_{-\beta}(x) & (x=1) .\end{cases}
$$

(The situations are sketched in Figure 4 for $i^{\prime}(1)=(1000)^{\infty}, i^{\prime}(1)=(2011)^{\infty}$ and $i^{\prime}(1)=$ $100(1)^{\infty}$.)
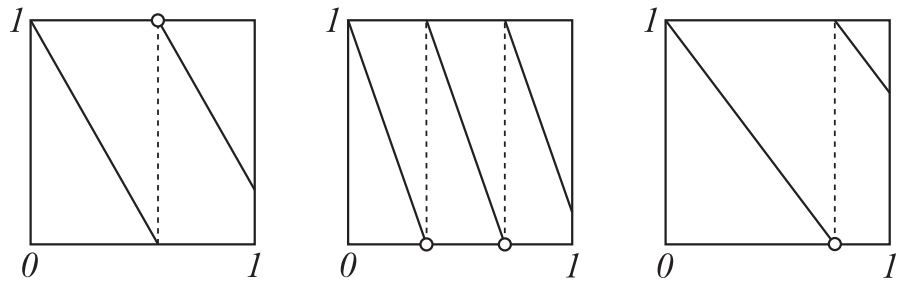

FIGURE 4. Graphs of $T_{-\beta}$ for $i^{\prime}(1)=(1000)^{\infty}$ (left), $i^{\prime}(1)=(2011)^{\infty}$ (center) and $i^{\prime}(1)=100(1)^{\infty}$ (right).

We define the alternating order $<_{\text {alt }}$ on $\{0,1, \ldots, b\}^{\mathbb{Z}_{+}}$by

$$
\left(\omega_{i}\right)<_{\text {alt }}\left(\omega_{i}^{\prime}\right) \text { if and only if } \omega_{i}=\omega_{i}^{\prime}(0 \leq i \leq n-1) \text { and } \begin{cases}\omega_{n}<\omega_{n}^{\prime} & (n \text { is even }), \\ \omega_{n}>\omega_{n}^{\prime} & (n \text { is odd })\end{cases}
$$

hold. We denote $\left(\omega_{i}\right) \leq_{\text {alt }}\left(\omega_{i}^{\prime}\right)$ if either $\left(\omega_{i}\right)<_{\text {alt }}\left(\omega_{i}^{\prime}\right)$ or $\left(\omega_{i}\right)=\left(\omega_{i}^{\prime}\right)$ holds. Let $\left(\Sigma_{-\beta}, \sigma\right)$ be the symbolic space of $\left([0,1], T_{-\beta}\right)$. Then following the proofs which appeared in $[\mathbf{1 2}$, Section 2] with a tiny modification, we have

$$
\Sigma_{-\beta}=\left\{\left(\omega_{i}\right): \sigma^{n}\left(\left(\omega_{i}\right)\right) \leq_{\text {alt }} i^{\prime}(1) \text { for } n \geq 0\right\} .
$$

We denote $i^{\prime}(1)=s_{0} s_{1} s_{2} \ldots$ and let $\Gamma_{-\beta}$ be a graph constructed by the following rules.

- There is an edge $V_{i} \stackrel{s_{i}}{\rightarrow} V_{i+1}$ for any $i \geq 0$.

- If $i$ is even, $0 \leq a \leq s_{i}-1$, and $s_{0} \cdots s_{j-1}$ is the suffix of maximal length of $s_{0} \cdots s_{i-1} a$, then there is an edge $V_{i} \stackrel{a}{\rightarrow} V_{j}$.

- If $i$ is odd, $s_{i}+1 \leq b \leq s_{0}$, and $s_{0} \cdots s_{j-1}$ is the suffix of maximal length of $s_{0} \cdots s_{i-1} b$, then there is an edge $V_{i} \stackrel{b}{\rightarrow} V_{j}$.

The situation is sketched in Figure 5 for $i^{\prime}(1)=100(1)^{\infty}$. Then by equation (3.1) and the definition of the alternating order, we have

$\Sigma_{-\beta}=\left\{\right.$ Set of all one-sided infinite paths started from some vertex $V_{i}$ of $\left.\Gamma_{-\beta}\right\}$.

Subsequently we will show the following proposition. 


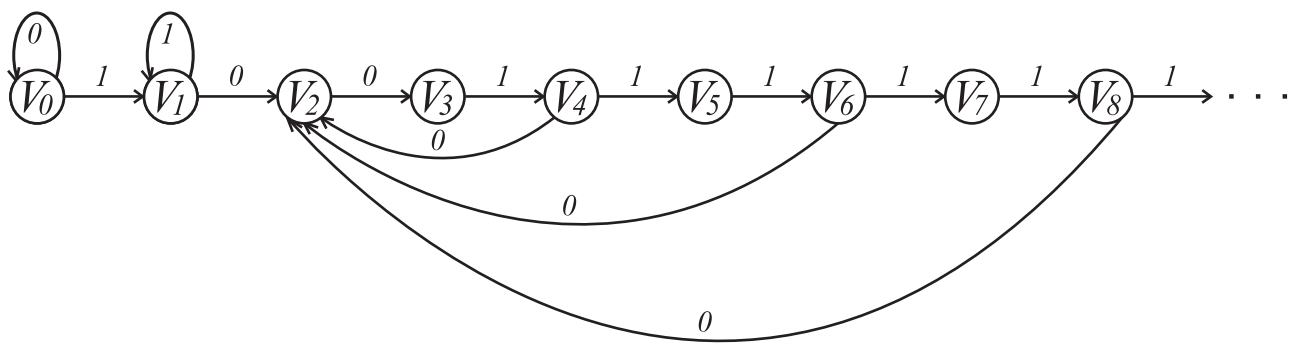

FIGURE 5. A graph $\Gamma_{-\beta}$ for $i^{\prime}(1)=100(1)^{\infty}$.

Proposition 3.4. Suppose that $i^{\prime}(1)$ is eventually periodic. Then $\left(\Sigma_{-\beta}, \sigma\right)$ satisfies the one-way specification property.

Proof. We denote $i^{\prime}(1)=s_{0} s_{1} s_{2} \cdots=t_{0} \cdots t_{u-1}\left(t_{u} \cdots t_{u+v-1}\right)^{\infty}$ for some $u \geq 0$ and $v \geq 1$. Since $i^{\prime}(1)=\lim _{x \rightarrow 1-0} i^{\prime}(x)=s_{0} s_{1} \cdots$, the cylinder set $\left[s_{0} \cdots s_{i}\right]$ is not a single point set for any $i \geq 0$. Thus, there is an edge $V_{i} \stackrel{a}{\rightarrow} V_{j}$ for some $i \geq u+v+2, j<i$ and $0 \leq a$ $\leq s_{0}$.

LEMMA 3.5. We have $j \leq u+v$. Moreover, for each $n \geq 0$, there is an edge $V_{i+2 n v} \stackrel{a}{\rightarrow} V_{j}$.

Proof. First we show $j \leq u+v$. By contradiction, suppose that $j \geq u+v+1$. Since there is an edge $V_{i} \stackrel{a}{\rightarrow} V_{j}, s_{0} \cdots s_{j-1}$ is the suffix of maximal length of $s_{0} \cdots s_{i-1} a$. Thus we can find a word $w^{\prime}$ such that $s_{0} \cdots s_{i-1} a=w^{\prime} s_{0} \cdots s_{j-1}$. Since $j \geq u+v+1$, we have $s_{j-1}=s_{j-v-1}$. Therefore, we have $a=s_{j-1}=s_{j-v-1}=s_{i-v}=s_{i}$, which contradicts with $s_{i} \neq a$.

Let $n \geq 0$. Since $s_{0} \cdots s_{j-1}$ is the suffix of $s_{0} \cdots s_{i-1} a$ and $j \leq u+v, s_{0} \cdots s_{j-1}$ is also the suffix of $s_{0} \cdots s_{i+2 n v-1} a$. In what follows we will show that $s_{0} \cdots s_{j-1}$ is the suffix of maximal length of $s_{0} \cdots s_{i+2 n v-1} a$. By contradiction, we assume that there exists a number $j^{\prime}>j$ such that $s_{0} \cdots s_{j^{\prime}-1}$ is the suffix of $s_{0} \cdots s_{i+2 n v-1} a$. Then $s_{0} \cdots s_{j}$ is the suffix of $s_{0} \cdots s_{i-1} a$. However, $s_{0} \cdots s_{j-1}$ is the suffix of maximal length of $s_{0} \cdots s_{i-1} a$. This is a contradiction.

For integers $0 \leq n \leq m$, let $\Gamma_{n, m}$ (respectively, $\Gamma_{n, \infty}$ ) be a subgraph of $\Gamma_{-\beta}$ from $V_{n}$ to $V_{m}$ (respectively, from $V_{n}$ ), that is, the vertexes of $\Gamma_{n, m}$ (respectively, $\Gamma_{n, \infty}$ ) are $V_{n}, V_{n+1}, \ldots, V_{m}$ (respectively, $V_{n}, V_{n+1}, \ldots$ ) and the edges of $\Gamma_{n, m}$ are the set of all edges of $\Gamma_{-\beta}$ from $V_{i}$ to $V_{j}$ for some $n \leq i, j \leq m$ (respectively, $i, j \geq n$ ). We set

$$
N:=\min \left\{k \geq 0: \Gamma_{k, \infty} \text { is irreducible }\right\} .
$$

By Lemma 3.5, such $N$ exists. We define sequences of integers $\left\{l_{i}\right\}$ and $\left\{n_{i}\right\}$ inductively as follows. First, we set $l_{1}:=0$ and

$$
n_{1}:=\max \left\{l_{1} \leq k<N: \Gamma_{l_{1}, k} \text { is irreducible }\right\} .
$$

Next, we set

$$
l_{2}:=\min \left\{k>n_{1}: \text { there are at least two edges into } V_{k}\right\}
$$


and

$$
n_{2}:=\left\{l_{2} \leq k<N: \Gamma_{l_{2}, k} \text { is irreducible }\right\} .
$$

Using this procedure inductively, we can construct integers $0=l_{1} \leq n_{1}<l_{2} \leq n_{2}<\cdots<$ $l_{q-1} \leq n_{q-1}<l_{q}=N<n_{q}=\infty$ such that:

- $\Gamma_{l_{i}, n_{i}}$ is irreducible for each $1 \leq i \leq q$;

- $V_{k} \stackrel{s_{k}}{\rightarrow} V_{k+1}$ is the only edge into $V_{k}$ for $n_{i} \leq k \leq l_{i+1}$ and $1 \leq i \leq q-1$;

- there is no edge from $V_{k}\left(k \notin\left\{l_{i}, \ldots, n_{i}\right\}\right)$ to $\Gamma_{l_{i}, n_{i}}$ except $V_{l_{i}-1} \stackrel{s_{l_{i}-1}}{\longrightarrow} V_{l_{i}}$ for $1 \leq i \leq q$.

In Figure 6, we sketch the situation for $i^{\prime}(1)=100(1)^{\infty}$.

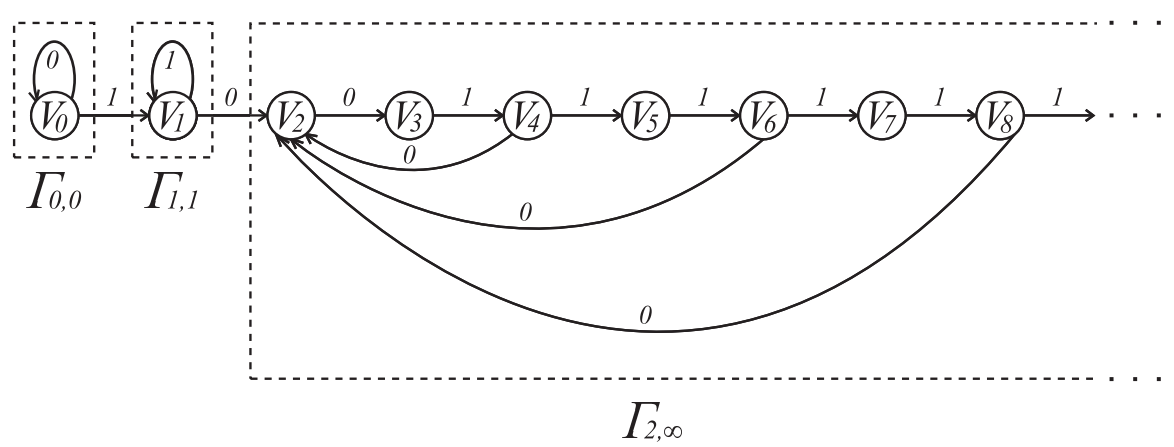

FIGURE 6. A graph representation of $\left(\Sigma_{-\beta}, \sigma\right)$ for $i^{\prime}(1)=100(1)^{\infty}$.

Let $X_{i}$ be a subshift generated by the graph $\Gamma_{l_{i}, n_{i}}$ for $1 \leq i \leq q$. More precisely, $X_{i}$ is the set of all infinite paths starting from some vertex in $\Gamma_{l_{i}, n_{i}}$. In what follows we will show that $\left(\Sigma_{-\beta}, \sigma\right)$ satisfies the one-way specification property with $\left\{X_{1}, \ldots, X_{q}\right\}$. Since $i^{\prime}(1)$ is eventually periodic, we can show that $X_{q}$ is sofic in a similar way to the proof of [11, Proposition 2]. Thus, $X_{i}$ is sofic and irreducible for each $1 \leq i \leq q$. Thus, $X_{i}$ satisfies the specification property, that is, there exists an integer $M \geq 0$ such that, for any $w_{1}, \ldots, w_{k} \in \mathcal{L}\left(X_{i}\right)$, we can find $v_{1}, \ldots, v_{k-1} \in \mathcal{L}\left(X_{i}\right)$ such that $\left|v_{j}\right| \leq M$ for $1 \leq j \leq k-1$ and $w_{1} v_{1} w_{2} v_{2} \cdots w_{k-1} v_{k-1} w_{k} \in \mathcal{L}\left(X_{i}\right)$ (see Proposition 2.5(1)). Therefore, $\left(\Sigma_{-\beta}, \sigma\right)$ satisfies the one-way specification property with $\left\{X_{1}, \ldots, X_{q}\right\}$.

Remark 3.6. As we mentioned in Section 1, our definition of the $(-\beta)$-transformation is slightly different from the original one in [12]. Ito and Sadahiro introduced a $(-\beta)$ transformation $R_{-\beta}$ on the interval $[-\beta /(\beta+1), 1 /(\beta+1))$ defined by $R_{-\beta}(x):=-\beta x-$ $\lfloor\beta /(\beta+1)-\beta x\rfloor$, where $\lfloor x\rfloor$ denotes the largest integer not exceeding a real number $x$. After that, Liao and Steiner [14] considered a $(-\beta)$-transformation $S_{-\beta}$ on the interval $(0,1]$ defined by $S_{-\beta}(x):=-\beta x+\lfloor\beta x\rfloor+1$, which is topologically conjugate to $R_{-\beta}$. Then, the differences between our $T_{-\beta}$ and Liao and Steiner's $S_{-\beta}$ are the following.

- $T_{-\beta}$ is defined on $[0,1]$, but $S_{-\beta}$ is defined on $(0,1]$.

- In Case 1 , that is, $i^{\prime}(1)$ is of the form $(w 0)^{\infty}$, then $T_{-\beta}(x)=0$ but $S_{-\beta}(x)=1$ for $x=1 / \beta, \ldots, b / \beta$. If we further assume that $\beta>1$ is an integer, then $T_{-\beta}(1)=0$ but $S_{-\beta}(1)=1$. 
The reason why we give a slightly modified version of the definition of the $(-\beta)$ transformation is the following.

- To formulate the large deviation principle, one usually requires that the space is Polish.

- By our definition of $T_{-\beta}, \Psi([w])$ is an interval with positive length for any $w \in$ $\mathcal{L}\left(\Sigma_{-\beta}\right)$, where $\Psi$ is a map defined in Section 2.3. If we define $T_{-\beta}$ as a natural extension of $S_{-\beta}$, then it may be that $\Psi([w])$ is a single point for some $w \in \Sigma_{-\beta}$.

We note that, in Case 2, $T_{-\beta}(x)=-\beta x+\lfloor\beta x\rfloor+1$ and so our definition of the ( $-\beta$ )-transformation $T_{-\beta}$ is a natural extension of $S_{-\beta}$. Therefore, the symbolic space $\left(\Sigma_{-\beta}, \sigma\right)$ of $\left([0,1], T_{-\beta}\right)$ coincides with that of $\left((0,1], S_{-\beta}\right)$ (and so also with that of $\left.\left([-\beta /(\beta+1), 1 /(\beta+1)), R_{-\beta}\right)\right)$.

\section{Proof of Theorem A}

In this section, we give a proof of Theorem A. First, we establish the upper bound. Since the metric entropy map is upper semicontinuous and $\varphi$ satisfies condition (1.1), it follows from [18, Theorems 3.1 and 3.2] that

$$
\limsup _{n \rightarrow \infty} \frac{1}{n} \log m\left(\left\{x \in X: \mathcal{E}_{n}(x) \in F\right\}\right) \leq \sup _{\mu \in F} q(\mu)
$$

holds for any closed set $F \subset \mathcal{M}(X)$.

To get the lower bound, it is sufficient to show that, for any $\mu \in \mathcal{M}_{f}(X)$ and any open neighborhood $G \subset \mathcal{M}(X)$ of $\mu$,

$$
\liminf _{n \rightarrow \infty} \frac{1}{n} \log m\left(\left\{x \in X: \mathcal{E}_{n}(x) \in G\right\}\right) \geq h(\mu)-\int \varphi d \mu
$$

holds. Let $\mu \in \mathcal{M}_{f}(X), G$ be an open neighborhood of $\mu$ and $\eta>0$. Choose $\zeta>0$ so small that $D(\mu, \nu) \leq 5 \zeta$ implies that $\nu \in G$ and $\left|\int \varphi d \mu-\int \varphi d \nu\right| \leq \eta$. Again by condition (1.1) there exist $\epsilon_{1}>0$ and $N_{1} \in \mathbb{N}$ such that, for any $0<\epsilon \leq \epsilon_{1}$, for any $n \geq N_{1}$, and for any $x \in X$,

$$
m\left(B_{n}(x, \epsilon)\right) \geq \exp \left(-\sum_{j=0}^{n-1} \varphi\left(f^{j}(x)\right)-n \eta\right)
$$

holds. We can also find $0<\epsilon_{2} \leq \epsilon_{1}$ such that $D\left(\delta_{x}, \delta_{y}\right) \leq \zeta$ whenever $d(x, y) \leq 2 \epsilon_{2}$.

On the other hand, by the ergodic decomposition theorem and the affinity of the entropy map, there exists $\lambda=\sum_{i=1}^{p} a_{i} \mu_{i}$ such that:

- the $\mu_{i}$ are ergodic;

- the $a_{i}$ are real numbers in $(0,1]$ such that $\sum_{i=1}^{p} a_{i}=1$;

- $D(\mu, \lambda) \leq \zeta$

- $h(\lambda)>h(\mu)-\eta$. 
By the assumption, $(X, f)$ satisfies the one-way specification property with some $\left\{X_{1}, \ldots, X_{q}\right\}$. Thus, it follows from Lemma 2.3 that, for each $1 \leq i \leq p$, we can find $1 \leq$ $j(i) \leq q$ such that $\mu_{i}\left(X_{j(i)}\right)=1$. Without loss of generality, we may assume $j(1) \leq j(2) \leq$ $\cdots \leq j(p)$. By Proposition 2.4, we can find $0<\epsilon \leq \epsilon_{2}$ and $N_{2} \geq N_{1}$ such that, for any $n \geq$ $N_{2}$ and any $1 \leq i \leq p$, there exists an $\left(n_{i}, 3 \epsilon\right)$-separated subset $\Gamma_{n, i} \subset \mathcal{E}_{n_{i}}^{-1}\left(\mathbb{B}\left(\mu_{i}, \zeta\right)\right) \cap X_{j(i)}$ such that

$$
\sharp \Gamma_{n, i} \geq \exp \left(n_{i}\left(h\left(\mu_{i}\right)-\eta\right)\right)
$$

holds. Here we set $\mathbb{B}\left(\mu_{i}, \eta\right):=\left\{v \in \mathcal{M}(X): D\left(\mu_{i}, v\right) \leq \eta\right\}$ and $n_{i}:=\max \left\{k \in \mathbb{Z}: a_{i} n \geq k\right\}$.

Let $M$ be as in the definition of the one-way specification property. Choose an integer $N \geq N_{2}$ so large that $(1 / N) \log (M+1)^{p-1} \leq \eta$ and

$$
\sum_{i=1}^{p}\left|a_{i}-\frac{n_{i}}{n_{1}+\cdots+n_{p}}\right| \leq \zeta
$$

hold. Fix any integer $n \geq N$. Then by the one-way specification property, for any $\mathbb{X}=$ $\left(x_{1}, \ldots, x_{p}\right) \in \prod_{i=1}^{p} \Gamma_{n, i}$, we can find $y(\mathbb{X}) \in X$ and integers $0 \leq M_{1}(\mathbb{X}), \ldots, M_{p-1}(\mathbb{X}) \leq$ $M$ such that

$$
d\left(f^{j}\left(x_{i}\right), f^{j+\sum_{t=1}^{i-1}\left(n_{t}+M_{t}(\mathbb{X})\right)}(y(\mathbb{X}))\right) \leq \epsilon \quad\left(0 \leq j \leq n_{i}-1,1 \leq i \leq p\right) .
$$

Since $0 \leq M_{i}(\mathbb{X}) \leq M$ for $1 \leq i \leq p$, it is easy to see that there exist integers $0 \leq$ $M_{1}, \ldots, M_{p-1} \leq M$ such that

$$
\sharp\left\{\mathbb{X} \in \prod_{i=1}^{p} \Gamma_{n, i}: M_{i}(\mathbb{X})=M_{i}, \quad 1 \leq i \leq p-1\right\} \geq \frac{1}{(M+1)^{p-1}} \sharp \prod_{i=1}^{p} \Gamma_{n, i} .
$$

For notational simplicity, we set

$$
\Gamma_{n}:=\left\{\mathbb{X} \in \prod_{i=1}^{p} \Gamma_{n, i}: M_{i}(\mathbb{X})=M_{i}, \quad 1 \leq i \leq p-1\right\} .
$$

Then we have

$$
\begin{aligned}
\sharp \Gamma_{n} & \geq \frac{1}{(M+1)^{p-1}} \sharp \prod_{i=1}^{p} \Gamma_{n, i} \\
& \geq \frac{1}{(M+1)^{p-1}} \prod_{i=1}^{p} \exp \left(n_{i}\left(h\left(\mu_{i}\right)-\eta\right)\right) \\
& \geq \frac{1}{(M+1)^{p-1}} \exp \left(\sum_{i=1}^{p} a_{i} n\left(h\left(\mu_{i}\right)-2 \eta\right)\right) \\
& \geq \frac{1}{(M+1)^{p-1}} \exp (n(h(\mu)-3 \eta)) \\
& \geq \exp (n(h(\mu)-4 \eta)) .
\end{aligned}
$$

We set $\tilde{n}:=\sum_{i=1}^{p-1}\left(n_{i}+M_{i}\right)+n_{p}$. Taking $N$ large if necessary, we may assume that

$$
D\left(\mathcal{E}_{n}(x), \sum_{i=1}^{p} \frac{n_{i}}{n_{1}+\cdots+n_{p}} \mathcal{E}_{n_{i}}\left(f^{\sum_{t=1}^{i-1}\left(n_{t}+M_{t}\right)}(x)\right)\right) \leq \zeta
$$

for $x \in X,((\tilde{n}-n) / n) \int \varphi d \mu \leq \eta,(2 \tilde{n} / n) \eta \leq 3 \eta$, and $\tilde{n} \geq \max \left\{N_{1}, N_{2}\right\}$ hold. 
LemmA 4.1. For any $\mathbb{X}, \mathbb{X}^{\prime} \in \Gamma_{n}$ with $\mathbb{X} \neq \mathbb{X}^{\prime}$, we have

$$
B_{\tilde{n}}(y(\mathbb{X}), \epsilon) \cap B_{\tilde{n}}\left(y\left(\mathbb{X}^{\prime}\right), \epsilon\right)=\emptyset .
$$

Proof. We denote $\mathbb{X}=\left(x_{1}, \ldots, x_{p}\right)$ and $\mathbb{X}^{\prime}=\left(x_{1}^{\prime}, \ldots, x_{p}^{\prime}\right)$. By $\mathbb{X} \neq \mathbb{X}^{\prime}$, we can find $1 \leq$ $i \leq p$ such that $x_{i} \neq x_{i}^{\prime}$. Since $x_{i}, x_{i}^{\prime} \in \Gamma_{n, i}$ and $\Gamma_{n, i}$ is $\left(n_{i}, 3 \epsilon\right)$-separated, there exists $0 \leq$ $j \leq n_{i}-1$ such that $d\left(f^{j}\left(x_{i}\right), f^{j}\left(x_{i}^{\prime}\right)\right)>3 \epsilon$. Thus, we have

$$
\begin{aligned}
d( & \left.f^{j+\sum_{t=1}^{i-1}\left(n_{t}+M_{t}\right)}(y(\mathbb{X})), f^{j+\sum_{t=1}^{i-1}\left(n_{t}+M_{t}\right)}\left(y\left(\mathbb{X}^{\prime}\right)\right)\right) \\
\geq & d\left(f^{j}\left(x_{i}\right), f^{j}\left(x_{i}^{\prime}\right)\right)-d\left(f^{j}\left(x_{i}\right), f^{j+\sum_{t=1}^{i-1}\left(n_{t}+M_{t}\right)}(y(\mathbb{X}))\right) \\
& -d\left(f^{j}\left(x_{i}^{\prime}\right), f^{j+\sum_{t=1}^{i-1}\left(n_{t}+M_{t}\right)}\left(y\left(\mathbb{X}^{\prime}\right)\right)\right) \\
> & 3 \epsilon-2 \epsilon \\
= & \epsilon
\end{aligned}
$$

which proves the lemma.

Lemma 4.2. Suppose that $\mathbb{X} \in \Gamma_{n}$ and $x \in B_{\tilde{n}}(y(\mathbb{X}), \epsilon)$. Then we have $D\left(\mu, \mathcal{E}_{n}(x)\right) \leq 5 \zeta$. In particular, $\mathcal{E}_{n}(x) \in G$ and $\left|\int \varphi d \mu-(1 / n) \sum_{j=0}^{n-1} \varphi\left(f^{j}(x)\right)\right| \leq \eta$ hold.

Proof. Denote $\mathbb{X}=\left(x_{1}, \ldots, x_{p}\right)$ and suppose that $x \in B_{\tilde{n}}(y(\mathbb{X}), \epsilon)$. Then we have

$$
d\left(f^{j}\left(x_{i}\right), f^{j+\sum_{t=1}^{i-1}\left(n_{t}+M_{t}\right)}(x)\right) \leq 2 \epsilon \quad\left(0 \leq j \leq n_{i}-1,1 \leq i \leq p\right) .
$$

Thus, it follows from $x_{i} \in \mathcal{E}_{n_{i}}^{-1}\left(\mathbb{B}\left(\mu_{i}, \zeta\right)\right)$ for $1 \leq i \leq p$ and Lemma 2.1 that

$$
\begin{aligned}
D\left(\mu, \mathcal{E}_{n}(x)\right) & \leq D\left(\sum_{i=1}^{p} a_{i} \mu_{i}, \sum_{i=1}^{p} \frac{n_{i}}{n_{1}+\cdots+n_{p}} \mathcal{E}_{n_{i}}\left(f^{\sum_{t=1}^{i-1}\left(n_{t}+M_{t}\right)}(x)\right)\right)+2 \zeta \\
& \leq D\left(\sum_{i=1}^{p} a_{i} \mu_{i}, \sum_{i=1}^{p} \frac{n_{i}}{n_{1}+\cdots+n_{p}} \mathcal{E}_{n_{i}}\left(x_{i}\right)\right)+3 \zeta \\
& \leq 5 \zeta
\end{aligned}
$$

which proves the lemma.

Now we continue the proof of Theorem A. By Lemmas 4.1 and 4.2, we have

$$
\begin{aligned}
\liminf _{n \rightarrow \infty} & \frac{1}{n} \log m\left(\left\{x \in X: \mathcal{E}_{n}(x) \in G\right\}\right) \\
\geq & \liminf _{n \rightarrow \infty} \frac{1}{n} \log m\left(\bigcup_{\mathbb{X} \in \Gamma_{n}} B_{\tilde{n}}(y(\mathbb{X}), \epsilon)\right) \\
\quad & \liminf _{n \rightarrow \infty} \frac{1}{n} \log \sum_{\mathbb{X} \in \Gamma_{n}} m\left(B_{\tilde{n}}(y(\mathbb{X}), \epsilon)\right) \\
\geq & \liminf _{n \rightarrow \infty} \frac{1}{n} \log \sharp \Gamma_{n} \exp \left(-\sum_{j=0}^{n-1} \varphi\left(f^{j}(y(\mathbb{X}))\right)-\tilde{n} \eta\right)
\end{aligned}
$$




$$
\begin{aligned}
& \geq \liminf _{n \rightarrow \infty} \frac{1}{n} \exp \left(n(h(\mu)-4 \eta)-\tilde{n} \int \varphi d \mu-2 \tilde{n} \eta\right) \\
& \geq h(\mu)-\int \varphi d \mu-8 \eta .
\end{aligned}
$$

This implies equation (4.1).

\section{Proof of Corollary 1}

The purpose of this section is to prove Corollary 1 stated in Section 2.3. For $\tilde{\mu} \in \mathcal{M}\left(\Sigma_{f}\right)$, we set $\hat{\Psi}(\tilde{\mu}):=\tilde{\mu} \circ \Psi^{-1}$. To prove Corollary 1 , we use the following lemmas.

LEMMA 5.1. The map $\hat{\Psi}: \mathcal{M}\left(\Sigma_{f}\right) \rightarrow \mathcal{M}([0,1])$ is continuous.

Proof. Suppose that $\left\{\tilde{\mu}_{n}\right\} \subset \mathcal{M}\left(\Sigma_{f}\right)$ and $\lim _{n \rightarrow \infty} \tilde{\mu}_{n}=\tilde{\mu}$. Then for any continuous function $\psi:[0,1] \rightarrow \mathbb{R}$, we have

$$
\begin{aligned}
\lim _{n \rightarrow \infty} \int \psi d \hat{\Psi}\left(\tilde{\mu}_{n}\right) & =\lim _{n \rightarrow \infty} \int \psi \circ \Psi d \tilde{\mu}_{n} \\
& =\int \psi \circ \Psi d \tilde{\mu} \\
& =\int \psi d \hat{\Psi}(\tilde{\mu}),
\end{aligned}
$$

which implies that $\hat{\Psi}$ is continuous.

Since $\left(\Sigma_{f}, \sigma\right)$ is a subshift, the entropy map is upper semicontinuous. So by Theorem A, and conditions (II) and (III), $\left(\Sigma_{f}, \sigma\right)$ satisfies a level-2 large deviation principle with $m_{f}$ and the rate function $\tilde{q}: \mathcal{M}\left(\Sigma_{f}\right) \rightarrow[-\infty, 0]$ is given by

$$
\tilde{q}(\mu)= \begin{cases}h(\mu)-\int \varphi \circ \Psi d \mu & \left(\mu \in \mathcal{M}_{\sigma}\left(\Sigma_{f}\right)\right), \\ -\infty & \text { (otherwise). }\end{cases}
$$

Let $q: \mathcal{M}([0,1]) \rightarrow[-\infty, 0]$ be a function given by (1.2).

LEMMA 5.2. For any subset $H \subset \mathcal{M}([0,1])$, we have

$$
\sup _{\tilde{\mu} \in \hat{\Psi}^{-1}(H)} \tilde{q}(\tilde{\mu})=\sup _{\mu \in H} q(\mu) .
$$

Proof. First we show the inequality $\sup _{\tilde{\mu} \in \hat{\Psi}^{-1}(H)} \widetilde{q}(\tilde{\mu}) \leq \sup _{\mu \in H} q(\mu)$. Let $\tilde{\mu} \in \hat{\Psi}^{-1}(H)$. Then it is sufficient to show that

$$
\widetilde{q}(\tilde{\mu}) \leq \sup _{\mu \in H} q(\mu) .
$$

If $\tilde{\mu}$ is not $\sigma$-invariant, then $\widetilde{q}(\tilde{\mu})=-\infty$ and so the inequality (5.1) is trivial. Thus, we assume that $\tilde{\mu}$ is $\sigma$-invariant. Since $\Psi$ is injective away from a countable set, we have $h(\tilde{\mu})=h(\hat{\Psi}(\tilde{\mu}))$. Thus, we have

$$
\begin{aligned}
\tilde{q}(\tilde{\mu}) & =h(\tilde{\mu})-\int \varphi \circ \Psi d \tilde{\mu} \\
& =h(\hat{\Psi}(\tilde{\mu}))-\int \varphi d \hat{\Psi}(\tilde{\mu})
\end{aligned}
$$




$$
\begin{aligned}
& =q(\hat{\Psi}(\tilde{\mu})) \\
& \leq \sup _{\mu \in H} q(\mu) .
\end{aligned}
$$

Here the last inequality follows from $\tilde{\mu} \in \hat{\Psi}^{-1}(H)$. Thus, we have (5.1).

Next, we show the opposite inequality. Let $\mu \in H$. It is sufficient to show that

$$
q(\mu) \leq \sup _{\tilde{\mu} \in \hat{\Psi}^{-1}(H)} \tilde{q}(\tilde{\mu})
$$

Without loss of generality, we may assume that $\mu$ is $f$-invariant. We set $\tilde{\mu}:=\mu \circ \Phi^{-1}$. Since $\Psi \circ \Phi$ is an identity map, we have $\hat{\Psi}(\tilde{\mu})=\mu$ and so $\tilde{\mu} \in \hat{\Psi}^{-1}(H)$. Moreover, since $\Phi$ is injective, we have $h(\mu)=h(\tilde{\mu})$. Thus, we have

$$
\begin{aligned}
q(\mu) & =h(\mu)-\int \varphi d \mu \\
& =h(\tilde{\mu})-\int \varphi \circ \Psi d \tilde{\mu} \\
& =\widetilde{q}(\tilde{\mu}),
\end{aligned}
$$

which implies (5.2).

Now, we prove Corollary 1. First we show that

$$
\liminf _{n \rightarrow \infty} \frac{1}{n} \log m\left(\left\{x \in[0,1]: \mathcal{E}_{n}(x) \in G\right\}\right) \geq \sup _{\mu \in G} q(\mu)
$$

for any open set $G \subset \mathcal{M}([0,1])$. Take any open set $G \subset \mathcal{M}([0,1])$. By Lemma 5.1, $\hat{\Psi}^{-1}(G)$ is open in $\mathcal{M}\left(\Sigma_{f}\right)$. Since $\left(\Sigma_{f}, \sigma\right)$ satisfies a level-2 large deviation principle with $m_{f}$ and the rate function is $\widetilde{q}$, we have

$$
\liminf _{n \rightarrow \infty} \frac{1}{n} \log m_{f}\left(\left\{\omega \in \Sigma_{f}: \mathcal{E}_{n}(\omega) \in \hat{\Psi}^{-1}(G)\right\}\right) \geq \sup _{\tilde{\mu} \in \hat{\Psi}^{-1}(G)} \tilde{q}(\tilde{\mu})=\sup _{\mu \in G} q(\mu),
$$

where we use Lemma 5.2 for the last equality. By condition (I) of Corollary 1, there exists a Borel subset $Y \subset \Sigma_{f}$ such that $[0,1] \backslash \Psi(Y)$ is countable and both $\Psi \circ \Phi:[0,1] \rightarrow[0,1]$ and $\Phi \circ \Psi: Y \rightarrow \Phi(\Psi(Y))$ are identity maps. Then we have

$$
\begin{aligned}
& \liminf _{n \rightarrow \infty} \frac{1}{n} \log m\left(\left\{x \in[0,1]: \mathcal{E}_{n}(x) \in G\right\}\right) \\
& \quad=\liminf _{n \rightarrow \infty} \frac{1}{n} \log m\left(\left\{x \in \Psi(Y): \mathcal{E}_{n}(x) \in G\right\}\right) \\
& \quad=\liminf _{n \rightarrow \infty} \frac{1}{n} \log m_{f}\left(\left\{\omega \in Y: \mathcal{E}_{n}(\omega) \in \hat{\Psi}^{-1}(G)\right\}\right) \\
& \quad=\liminf _{n \rightarrow \infty} \frac{1}{n} \log m_{f}\left(\left\{\omega \in \Sigma_{f}: \mathcal{E}_{n}(\omega) \in \hat{\Psi}^{-1}(G)\right\}\right) .
\end{aligned}
$$

Thus we get the lower bound. The upper bound can be shown similarly.

\section{Applications}

In this section, we apply Theorem A and Corollary 1 to systems which appeared in Section 3. 


\subsection{Simple symbolic example}

Proposition 6.1. Let $(I, f)$ be as in Example 3.1. Then $(I, f)$ satisfies a level-2 large deviation principle with the Lebesgue measure $m$ on $[0,1]$, and the rate function $q: \mathcal{M}[0,1] \rightarrow[-\infty, 0]$ is given by

$$
q(\mu)= \begin{cases}h(\mu)-\log 3 & (\mu \text { is invariant }), \\ -\infty & (\text { otherwise })\end{cases}
$$

Proof. Let $\left(\Sigma_{f}, \sigma\right)$ be the symbolic space of $(I, f)$. We set $J_{0}=\left[0, \frac{1}{6}\right), J_{1}=\left[\frac{1}{6}, \frac{1}{2}\right), J_{2}=$ $\left[\frac{1}{2}, \frac{2}{3}\right), J_{3}=\left[\frac{2}{3}, \frac{5}{6}\right)$, and $J_{4}=\left[\frac{5}{6}, 1\right]$. Define a measurable map $\Phi: I \rightarrow \Sigma_{f}$ by

$$
(\Phi(x))_{i}=j \text { if and only if } f^{i}(x) \in J_{j} .
$$

Then it is not difficult to see that $\Phi$ satisfies (1) of Corollary 1.

Thus, it is sufficient to show that $\left(\Sigma_{f}, \sigma\right)$ satisfies conditions (II) and (III) of Corollary 1 for $m_{f}=m \circ \Phi^{-1}$ and the constant function $\varphi \equiv \log 3$. As we have shown in Example 3.1, $\left(\Sigma_{f}, \sigma\right)$ satisfies the one-way specification property, and so (III) holds. Since the slope of $f$ is 3 , and the definition of $\Phi$, we have

$$
\left(\frac{1}{2}\right) 3^{-n} \leq m \circ \Phi^{-1}([w]) \leq 3^{-n} .
$$

Thus, it follows from Proposition 2.5(3) that condition (1.1) holds for $m_{f}$ and $\varphi \circ \Psi \equiv \log 3$. Thus we have (II) and so the proposition is proved.

\subsection{Axiom A diffeomorphisms: proof of Theorem B}

In this subsection, we will prove Theorem B by showing the following two propositions.

Proposition 6.2. Let $f: S^{2} \rightarrow S^{2}$ be a Smale's horseshoe map given in Example 3.2. Then $\left(S^{2}, f\right)$ satisfies a level-2 large deviation principle with the normalized Lebesgue measure $m$ on $S^{2}$ and the rate function $q: \mathcal{M}\left(S^{2}\right) \rightarrow[-\infty, 0]$ is given by (1.2) for some $\varphi \in C\left(S^{2}\right)$.

Proof. First, as we have shown in Example 3.2, $\left(S^{2}, f\right)$ satisfies the one-way specification property. Since $f$ is $C^{\infty}$, the metric entropy map is upper semicontinuous (see [16] for instance). Let $\Lambda$ be a hyperbolic set as in (S6) and $U_{\epsilon}$ be an $\epsilon$-neighborhood of the nonwandering set $\Omega(f)=\left\{p_{\infty}\right\} \cup \Lambda \cup\left\{p_{0}\right\}$ for $\epsilon>0$. Then it is easy to see that, for any $\epsilon>0$, we can find an integer $N>0$ such that

$$
\sharp\left\{n \geq 0: f^{n}(x) \notin U_{\epsilon}\right\} \leq N
$$

holds for any $x \in S^{2}$. This enables us to find $\varphi \in C\left(S^{2}\right)$ satisfying (1.1) and

$$
\varphi(x)= \begin{cases}\log \left|\operatorname{Jac}\left(D_{x} f\right)\right| & \left(x=p_{\infty}\right), \\ \log \lambda_{u} & (x \in \Lambda), \\ 0 & \left(x=p_{0}\right),\end{cases}
$$

where $\operatorname{Jac}\left(D_{x} f\right)$ stands for the Jacobian of $D_{x} f$ and $\lambda_{u}>2$ is a constant as in (S2) of Example 3.2. So the proposition follows from Theorem A. 
Proposition 6.3. Let $f: \mathbb{T}^{2} \rightarrow \mathbb{T}^{2}$ be a DA map given in Example 3.3. Then $\left(\mathbb{T}^{2}, f\right)$ satisfies a level-2 large deviation principle with the normalized Lebesgue measure $m$ on $\mathbb{T}^{2}$ and the rate function $q: \mathcal{M}\left(\mathbb{T}^{2}\right) \rightarrow[-\infty, 0]$ is given by (1.2) for some $\varphi \in C\left(\mathbb{T}^{2}\right)$.

Proof. Since $f$ is $C^{\infty}$, the entropy map is upper semicontinuous. Let $\Lambda$ be a hyperbolic set as in Example 3.3 and $U_{\epsilon}$ be an $\epsilon$-neighborhood of the non-wandering set $\Omega(f)=\left\{p_{0}\right\} \cup \Lambda$ for $\epsilon>0$. Then it follows from (D3) of Example 3.3 that, for any $\epsilon>0$, we can find an integer $N>0$ such that

$$
\sharp\left\{n \geq 0: f^{n}(x) \notin U_{\epsilon}\right\} \leq N
$$

holds for any $x \in \mathbb{T}^{2}$. So by the standard volume lemma on $\Lambda[2,(4.7)]$, we can find a continuous function $\varphi$ on $\mathbb{T}^{2}$ so that

$$
\varphi(x)= \begin{cases}\log \left|\operatorname{Jac}\left(D_{x} f\right)\right| & \left(x=p_{0}\right), \\ \log \left\|\left.D_{x} f\right|_{E^{u}(x)}\right\| & (x \in \Lambda)\end{cases}
$$

and condition (1.1) hold. Here $E^{u}$ denotes the unstable bundle on $\Lambda$. Moreover, we have already shown that $\left(\mathbb{T}^{2}, f\right)$ satisfies the one-way specification property with $\left\{\left\{p_{0}\right\}, \Lambda\right\}$ in Example 3.3. So, by Theorem A, we have the proposition.

Theorem B is a direct consequence of Propositions 6.2 and 6.3.

\section{3. (- $\beta$ )-transformations: proof of Theorem $C$}

The aim of this subsection is to give a proof of Theorem C. Let $\beta>1$ be a Yrrap number. First, we define a map $\Phi:[0,1] \rightarrow \Sigma_{-\beta}$ as follows. If $i^{\prime}(1)=(\cdots 0)^{\infty}$, then let $J_{0}=$ $[0,1 / \beta], J_{i}=(i / \beta,(i+1) / \beta]$ for $1 \leq i \leq b-1$, and $J_{b}=(b / \beta, 1]$. If $i^{\prime}(1)$ is not of the form $(\cdots 0)^{\infty}$, then let $J_{i}=[i / \beta,(i+1) / \beta)$ for $0 \leq i \leq b-1$, and $J_{b}=[b / \beta, 1]$. Then, we define $\Phi:[0,1] \rightarrow \Sigma_{-\beta}$ by $(\Phi(x))_{i}=j$ if and only if $T_{-\beta}(x) \in J_{j}$. Then, it is not difficult to see that $\Phi$ satisfies (I) of Corollary 1. It follows from 3.4 that $\left(\Sigma_{-\beta}, \sigma\right)$ satisfies the one-way specification property. So, by Corollary 1 and Proposition 2.5(3), it is sufficient to show that

$$
\limsup _{n \rightarrow \infty} \sup _{w \in \mathcal{L}_{n}\left(\Sigma_{-\beta}\right)}\left(\frac{1}{n} \log m_{-\beta}([w])+\log \beta\right) \leq 0
$$

and

$$
\liminf _{n \rightarrow \infty} \inf _{w \in \mathcal{L}_{n}\left(\Sigma_{-\beta}\right)}\left(\frac{1}{n} \log m_{-\beta}([w])+\log \beta\right) \geq 0 .
$$

Here we set $m_{-\beta}:=m \circ \Phi^{-1}$ with the Lebesgue measure $m$ on $[0,1]$.

First, we show equation (6.3). For $w \in \mathcal{L}_{n}\left(\Sigma_{-\beta}\right)$, it is easy to see that $\Phi^{-1}([w])$ is a sub-interval of $(0,1]$ and $T_{-\beta}^{n}: \Phi^{-1}([w]) \rightarrow(0,1]$ is a continuous linear map whose slope is equal to $\beta^{n}$. Thus we have

$$
m_{-\beta}([w])=m\left(\Phi^{-1}([w])\right) \leq \beta^{-n} .
$$

This implies (6.3). In what follows we will show equation (6.4).

LeMmA 6.4. Let $w \in \mathcal{L}_{n}\left(\Sigma_{-\beta}\right)$ and suppose that there exist $0 \leq c<d \leq b$ such that $w c, w d \in \mathcal{L}\left(\Sigma_{-\beta}\right)$. Then

$$
m_{-\beta}([w]) \geq\left(1-\frac{b}{\beta}\right) \beta^{-n}
$$


Proof. It is easy to see that if $\left(a_{1}, a_{2}\right) \subset J_{i}$ and there exists a point $b_{1} \in J_{j}$ such that $T_{-\beta}\left(b_{1}\right)=a_{1}$, then there exists a point $b_{2} \in J_{j}$ such that $T_{-\beta}\left(b_{2}\right)=a_{2}$ and $\beta\left(b_{1}-b_{2}\right)=$ $a_{2}-a_{1}$. Thus, for any $x \in \Phi^{-1}([w])$,

$$
\left.T_{-\beta}^{-1}\left(\cdots\left(T_{-\beta}^{-1}\left(T_{-\beta}^{-1}\left(w_{n} \beta^{-1}, T_{-\beta}^{n-1}(x)\right) \cap J_{w_{n-1}}\right) \cap J_{w_{n-2}}\right) \cap \cdots\right) \cap J_{w_{1}}\right)
$$

is an interval of length $\beta^{-n+1}\left|T_{-\beta}^{n-1}(x)-w_{n} \beta^{-1}\right|$ and is contained in $\Phi^{-1}([w])$. So we have

$$
m_{-\beta}([w]) \geq \beta^{-n+1}\left|T_{-\beta}^{n-1}-w_{n} \beta^{-1}\right|
$$

for any $x \in \Phi^{-1}([w])$. On the other hand, $T_{-\beta}\left(w_{n} \beta^{-1}\right)=1$ and so $w b \in \Sigma_{-\beta}$. By the assumption, there exists a point $x \in \Phi^{-1}([w])$ such that $T_{-\beta}^{n}(x) \notin J_{b}$. Then we have

$$
\left|T_{-\beta}^{n}(x)-T_{-\beta}\left(w_{n} \beta^{-1}\right)\right| \geq\left|J_{b}\right|=\left(1-\frac{b}{\beta}\right),
$$

which implies

$$
\left|T_{-\beta}^{n-1}(x)-w_{n} \beta^{-1}\right| \geq \beta^{-1}\left(1-\frac{b}{\beta}\right) .
$$

Combining equations (6.6) and (6.7), we have (6.5).

For $w \in \mathcal{L}\left(\Sigma_{-\beta}\right)$, let $g_{\beta}(w)$ be a minimum integer $i \geq 0$ so that there exists $v \in \mathcal{L}_{i}\left(\Sigma_{-\beta}\right)$ and integers $0 \leq c<d \leq b$ such that $w v c, w v d \in \mathcal{L}\left(\Sigma_{-\beta}\right)$, and set

$$
g_{\beta}(n):=\sup _{w \in \mathcal{L}_{n}\left(\Sigma_{-\beta}\right)} g_{\beta}(w)
$$

for $n \geq 1$.

LemMA 6.5. Suppose that $\lim _{n \rightarrow \infty}\left(g_{\beta}(n) / n\right)=0$. Then equation (6.4) holds.

Proof. Let $\epsilon>0$. By the assumption, there exists an integer $N$ such that, for any $n \geq N$, $g_{\beta}(n) \leq n \epsilon$ holds. Fix any $n \geq N$ and $w \in \mathcal{L}_{n}\left(\Sigma_{-\beta}\right)$. Then we have $g_{\beta}(w) \leq g_{\beta}(n) \leq n \epsilon$. Thus we can find $v \in \mathcal{L}\left(\Sigma_{-\beta}\right)$ and $0 \leq c<d \leq b$ such that

$$
|v| \leq n \epsilon \quad \text { and } \quad w v c, w v d \in \mathcal{L}\left(\Sigma_{-\beta}\right)
$$

holds. So it follows from Lemma 6.4 that

$$
\begin{aligned}
m_{-\beta}([w]) & \geq m_{-\beta}([w v]) \\
& \geq\left(1-\frac{b}{\beta}\right) \beta^{-|w v|} \\
& \geq\left(1-\frac{b}{\beta}\right) \beta^{-n(1+\epsilon)} .
\end{aligned}
$$

Since $w \in \mathcal{L}_{n}\left(\Sigma_{-\beta}\right), n \geq N$, and $\epsilon>0$ are arbitrary, the above inequality implies (6.4).

Thus, it follows from Lemmas 3.5 and 6.5 that if $\beta>1$ is Yrrap, then equation (6.4) holds. Therefore, Theorem $\mathrm{C}$ is proved. 
Example 6.6. Finally, we give an example of the $(-\beta)$-transformation whose rate functions with respect to the Lebesgue measure and maximal entropy measure do not coincide. Let $\beta>1$ be the minimal Pisot number, that is, the real root of $X^{3}-X-1=0$. Then it is easy to see that $i^{\prime}(1)=100(1)^{\infty}$, and so $\beta$ is a Yrrap number and $\left(\Sigma_{-\beta}, \sigma\right)$ is generated by a graph $\Gamma_{-\beta}$, which is shown in Figure 5. Thus, we have

$$
\mathcal{M}_{\sigma}\left(\Sigma_{-\beta}\right)=\left\{\delta_{0} \infty\right\} \cup\left\{\delta_{1} \infty\right\} \cup \mathcal{M}_{\sigma}\left(X_{3}\right) .
$$

Here $X_{3}$ is a subshift generated by a graph $\Gamma_{2, \infty}$, shown in Figure 6. In particular, we can see that $\mathcal{M}_{\sigma}^{e}\left(\Sigma_{-\beta}\right)$ is not dense in $\mathcal{M}_{\sigma}\left(\Sigma_{-\beta}\right)$. It follows from [14, Corollary 2.4] that $T_{-\beta}$ (respectively, $\left(\Sigma_{-\beta}, \sigma\right)$ ) admits a unique measure of maximal entropy $v$ (respectively, $\nu_{-\beta}$ ). Then by (6.8), $\nu_{-\beta}$ is supported on $X_{3}$. Since $\left(X_{3}, \sigma\right)$ satisfies the specification property, by Theorem 1.1 with an easy additional calculation, we can show that $\left(\Sigma_{-\beta}, \sigma\right)$ satisfies a level-2 large deviation principle with $\nu_{-\beta}$ and the rate function $q_{v_{-\beta}}: \mathcal{M}\left(\Sigma_{-\beta}\right) \rightarrow[-\infty, 0]$ is given by

$$
q_{v_{-\beta}}(\mu)= \begin{cases}h(\mu)-\log \beta & \left(\mu \in \mathcal{M}_{\sigma}\left(X_{3}\right)\right), \\ -\infty & \text { (otherwise) } .\end{cases}
$$

So in a similar way to the proof of Corollary 1 , we can show that $\left([0,1], T_{-\beta}\right)$ satisfies a level-2 large deviation principle with $v$ and the rate function $q_{v}: \mathcal{M}([0,1]) \rightarrow[-\infty, 0]$ is given by

$$
q_{\nu}(\mu)= \begin{cases}h(\mu)-\log \beta & \left(\mu \in \hat{\Psi}^{-1}\left(\mathcal{M}_{\sigma}\left(X_{3}\right)\right)\right), \\ -\infty & \text { (otherwise) }\end{cases}
$$

Here we set $\hat{\Psi}(\mu):=\mu \circ \Psi^{-1}$ for $\mu \in \mathcal{M}\left(\Sigma_{-\beta}\right)$ and $\Psi: \Sigma_{-\beta} \rightarrow[0,1]$ is as in Section 2.3. On the other hand, by Theorem $\mathrm{C},\left([0,1], T_{-\beta}\right)$ satisfies a level-2 large deviation principle with the Lebesgue measure $m$ and the rate function $q_{m}: \mathcal{M}([0,1]) \rightarrow[-\infty, 0]$ is given by (1.3). Thus, we have $q_{v}(\mu) \neq q_{m}(\mu)$ for $\mu \in \mathcal{M}_{T_{-\beta}}([0,1]) \backslash \hat{\Psi}^{-1}\left(\mathcal{M}_{\sigma}\left(X_{3}\right)\right)$. Moreover, it is not difficult to see that

$$
\begin{aligned}
& \mathcal{M}_{T_{-\beta}}([0,1]) \backslash \hat{\Psi}^{-1}\left(\mathcal{M}_{\sigma}\left(X_{3}\right)\right) \\
& \quad=\left\{a \delta_{1 /(\beta+1)}+(1-a) \mu: 0<a \leq 1, \mu \in \mathcal{M}_{T_{-\beta}}([0,1])\right\} .
\end{aligned}
$$

In particular, this set is not empty. Thus, we conclude that $q_{v} \neq q_{m}$.

Acknowledgement. The author wishes to express his deepest appreciation to the referee for his/her careful reading of the manuscript and critical comments. This work was supported by JSPS KAKENHI grant number JP16K17611.

\section{REFERENCES}

[1] R. Bowen. Periodic points and measures for Axiom A diffeomorphisms. Trans. Amer. Math. Soc. 154 (1971), 377-397.

[2] R. Bowen. Equilibrium States and the Ergodic Theory of Anosov Diffeomorphisms (Lecture Notes in Mathematics, 470), 2nd edn, with a preface by David Ruelle. Ed. J.-R. Chazottes. Springer, Berlin, 2008.

[3] Y. M. Chung. Large deviations on Markov towers. Nonlinearity 24 (2011), 1229-1252.

[4] Y. M. Chung and H. Takahasi. Large deviation principle for Benedicks-Carleson quadratic maps: Comm. Math. Phys. 315 (2012) 803-826. 
[5] V. Climenhaga and D. J. Thompson. Intrinsic ergodicity beyond specification: $\beta$-shifts, $S$-gap shifts, and their factors. Israel J. Math. 192(2) (2012), 785-817.

[6] V. Climenhaga, D. J. Thompson and K. Yamamoto. Large deviations for systems with non-uniform structure. Trans. Amer. Math. Soc. 369(6) (2017), 4167-4192.

[7] K. Dajani and S. D. Ramawadh. Symbolic dynamics of $(-\beta)$-expansions. J. Integer Seq. 15(2) (2012), Article 12.2.6.

[8] M. Dateyama. The almost weak specification property for ergodic group automorphisms of abelian groups. J. Math. Soc. Japan 42(2) (1990), 341-351.

[9] M. Denker, C. Grillenberger and K. Sigmund. Ergodic Theory on Compact Spaces (Lecture Notes in Mathematics, 527). Springer, Berlin, 1976.

[10] A. Eizenberg, Y. Kifer and B. Weiss. Large deviations for $\mathbb{Z}^{d}$-actions. Comm. Math. Phys. 164 (1994), 433-454.

[11] C. Frougny and A. C. Lai. On negative bases, Proc. of DLT 09 (Lecture Notes in Computer Science, 5583) Springer, Berlin, 2009, pp. 252-263.

[12] S. Ito and T. Sadahiro. Beta-expansions with negative bases. Integers 9 (2009), A22, 239-259.

[13] D. Kwietniak, M. Lacka and P. Oprocha. A panorama of specification-like properties and their consequences. Dynamics and Numbers (Contemporary Mathematics, 669). American Mathematical Society, Providence, RI, 2016, pp. 155-186.

[14] L. Liao and W. Steiner. Dynamical properties of the negative beta transformation. Ergod. Th. Dynam. Sys. 32 (2012), 1673-1690.

[15] B. Marcus. A note on periodic points for ergodic toral automorphisms. Monatsh. Math. 89(2) (1980), 121-129.

[16] S. E. Newhouse. Continuity properties of entropy. Ann. of Math. (2) 129(2) (1989), 215-235.

[17] W. Parry. On the $\beta$-expansions of real numbers. Act Math. Hung. 11 (1960), 401-416.

[18] C.-E. Pfister and W. G. Sullivan. Large deviations estimates for dynamical systems without the specification property. Application to the $\beta$-shifts. Nonlinearity 18 (2005), 237-261.

[19] C.-E. Pfister and W. G. Sullivan. On the topological entropy of saturated sets. Ergod. Th. Dynam. Sys. 27(3) (2007), 929-956.

[20] A. Renyi. Representations for real numbers and their ergodic properties. Acta Math. Acad. Sci. Hungar. 8 (1957), 477-493.

[21] C. Robinson. Dynamical systems. Stability, Symbolic Dynamics, and Chaos, 2nd edn. CRC Press, London, 1999.

[22] Y. Takahashi. Entropy functional (free energy) for dynamical systems and their random perturbations. Stochastic analysis (Katata/Kyoto, 1982) (North-Holland Mathematical Library, 32). North-Holland, Amsterdam, 1984, pp. 437-467.

[23] D. J. Thompson. Irregular sets, the $\beta$-transformation and the almost specification property. Trans. Amer. Math. Soc. 364(10) (2012), 5395-5414.

[24] P. Varandas. Non-uniform specification and large deviations for weak Gibbs measures. J. Stat. Phys. 146(2) (2012), 330-358.

[25] K. Yamamoto. On the weaker forms of the specification property and their applications. Proc. Amer. Math. Soc. 137(11) (2009), 3807-3814.

[26] L.-S. Young. Some large deviation results for dynamical systems. Trans. Amer. Math. Soc. 318(2) (1990), 525-543.

\author{
Kenichiro Yamamoto \\ Department of General Education \\ Nagaoka University of Technology \\ Niigata 940-2188 \\ Japan
}

(E-mail:k_yamamoto@vos.nagaokaut.ac.jp) 